\title{
Functional Subpopulations of V3 Interneurons in the Mature Mouse Spinal Cord
}

\author{
Joanna Borowska, ${ }^{1}$ Christopher T. Jones, ${ }^{2}$ Han Zhang, ${ }^{1}$ Jake Blacklaws, ${ }^{1}$ Martyn Goulding, ${ }^{3}$ and Ying Zhang ${ }^{1}$ \\ Departments of ${ }^{1}$ Medical Neuroscience, and ${ }^{2}$ Mathematics and Statistics, Dalhousie University, Halifax, Nova Scotia B3H 4R2, Canada, and ${ }^{3}$ Molecular \\ Neurobiology Laboratory, the Salk Institute for Biological Studies, La Jolla, California 92037
}

V3 interneurons (INs) are a major group of excitatory commissural interneurons in the spinal cord, and they are essential for producing a stable and robust locomotor rhythm. V3 INs are generated from the ventral-most progenitor domain, p3, but migrate dorsally and laterally during postmitotic development. At birth, they are located in distinctive clusters in the ventral horn and deep dorsal horn. To assess the heterogeneity of this genetically identified group of spinal INs, we combined patch-clamp recording and anatomical tracing with cluster analysis. We examined electrophysiological and morphological properties of mature V3 INs identified by their expression of tdTomato fluorescent proteins in Sim $1^{\text {Cre/+}}$; Rosa $a^{\text {floxstop26TdTom }}$ mice. We identified two V3 subpopulations with distinct intrinsic properties and spatial distribution patterns. Ventral V3 INs, primarily located in lamina VIII, possess a few branching processes and were capable of generating rapid tonic firing spikes. By contrast, dorsal V3 INs exhibited a more complex morphology and relatively slow average spike frequency with strong adaptation, and they also displayed large sag voltages and post-inhibitory rebound potentials. Our data suggested that hyperpolarization-activated cation channel currents and T-type calcium channel currents may account for some of the membrane properties of V3 INs. Finally, we observed that ventral and dorsal V3 INs were active in different ways during running and swimming, indicating that ventral V3 INs may act as premotor neurons and dorsal V3 INs as relay neurons mediating sensory inputs. Together, we detected two physiologically and topographically distinct subgroups of V3 INs, each likely playing different roles in locomotor activities.

\section{Introduction}

Various groups of neurons in the spinal cord form neural circuits, known as locomotor central pattern generators (CPGs). They are responsible for generating the basic rhythmic activity in limb muscles that occurs during locomotion (Grillner and Wallén, 1985; Kjaerulff and Kiehn, 1996). Much effort has been devoted to defining the specific roles of individual spinal interneurons (INs) in CPGs (Jankowska, 2001). The discoveries describing the genetic origins of spinal neurons have provided powerful tools to identify different neuronal classes according to their molecular profiles (Goulding, 2009). More recent work, however, has demonstrated that many of these genetically identified populations are still heterogeneous. Several groups (V0, V1, and V2) can be further subdivided based on the expression of downstream transcription factors (Arber, 2012), with each of these subpopulations

\footnotetext{
Received April 30, 2013; revised 0ct. 11, 2013; accepted 0ct. 23, 2013

Author contributions: Y.Z. designed research; J. Borowska, H.Z., and J. Blacklaws performed research; M.G. contributed unpublished reagents/analytic tools; J. Borowska, C.T.J., H.Z., and Y.Z. analyzed data; C.T.J. and Y.Z. wrote the paper.

This work was supported by the Canadian Institute of Health Research (Grant MOP-110950 to Y.Z.) and Natural Sciences and Engineering Research Council of Canada (Grant 38620 to Y.Z.) and by the National Institutes of Health (Grants P01 NS031249 and R37 NS037075 to M.G.). We thank Dr. Hongkui Zeng for providing the Rosa floxstop26TdTom mouse, and Dr. Guisheng Zhong for assisting on the data analyses. We also thank Drs. Simon Gosgnach, Tuan Bui, and Kazue Semba for helpful discussions of this manuscript; and Stephen Whitefield for his technical support.

The authors declare no competing financial interests.

Correspondence should be addressed to Ying Zhang, Department of Medical Neuroscience, Dalhousie University, Halifax, NS B3H 4R2, Canada. E-mail: ying.zhang@dal.ca.

DOI:10.1523/JNEUROSCI.2005-13.2013

Copyright $\odot 2013$ the authors $\quad 0270-6474 / 13 / 3318553-13 \$ 15.00 / 0$
}

potentially having unique functions in motor control. V3 INs are defined by the expression of the Sim1 transcription factor and comprise a major group of excitatory commissural neurons in the ventral spinal cord (Zhang et al., 2008). They originate from the most ventral progenitor domain, $\mathrm{p} 3$, where the transcription factor Nkx2.2 is expressed (Briscoe et al., 1999; Sugimori et al., 2007). Genetic suppression of the activity of the entire V3 population in mice causes animals to exhibit unstable and uncoordinated gaits (Zhang et al., 2008). The precise function of V3 INs in the motor system, however, is still not understood. It was shown that V3 INs were not only located in different regions of the ventral spinal cord, but also spread into deep dorsal horn in the lower thoracic and upper lumbar segments (Geiman et al., 2006). Such a distribution pattern raises the possibility that V3 INs at different locations of the spinal cord represent distinct functional subpopulations.

Although no known molecular markers are currently available to distinguish between V3 IN subtypes, we believe that investigating their intrinsic properties could provide another means of defining the unique identity of these cells. Membrane properties and morphological structures have been used extensively to classify neuron groups in the cortex and other regions of the CNS (Ascoli et al., 2008; Graves et al., 2012). Interneurons in the spinal cord have also shown to have specific intrinsic properties that vary based on function. For example, Renshaw cells, a subset of the V1 INs (Stam et al., 2012; Alvarez et al., 2013), mediate the recurrent inhibition to motor neurons and have unique anatomical and physiological characteristics (Renshaw, 
1941, 1946). In a recent study, dI6 INs that are rhythmically active during locomotion could be divided into two groups based on unique intrinsic properties, suggesting divergent functions during behavior (Dyck et al., 2012). Such results suggest that specific membrane properties are closely related to neuronal functions in the spinal cord.

In this study, we have investigated electrophysiological and morphological properties of mature V3 INs in the mouse spinal cord and demonstrated that two spatially and functionally distinct V3 subpopulations exist. This work has established a solid foundation to further comprehend the specific roles of different V3 subpopulations play in locomotor activities.

\section{Materials and Methods}

\section{Mouse strains}

The generation and genotyping of $\mathrm{Sim} 1^{\mathrm{Cre} /+}$ mice was described previously by Zhang et al. (2008). Rosa floxstop26TdTom mice (Madisen et al., 2010; stock \#007908, The Jackson Laboratory) were provided by Dr. Hongkui Zeng of the Allen Institute for Brain Science (Seattle, WA). Sim $1^{\mathrm{Cre} /+}$; Rosa floxstop $26 \mathrm{TdTom}$ mice were generated by crossing these two strains. All procedures were performed in accordance with the Canadian Council on Animal Care and approved by the University Committee on Laboratory Animals at Dalhousie University.

\section{Electrophysiological experiments}

Slice preparations. Electrophysiological experiments were carried out on weaned, juvenile postnatal day 20 (P20)-P23 Sim ${ }^{\mathrm{Cre} /+}$; Rosa $a^{\text {floxstop26TdTom }}$ mice, both males and females. Mice were anesthetized by intraperitoneal injection of a mixture of ketamine $(60 \mathrm{mg} / \mathrm{kg})$ and xylazine $(12 \mathrm{mg} / \mathrm{kg})$. After they lost their righting reflex, mice were cooled in an ice bed and decapitated. The spinal cord lumbar region (T13-L3) was dissected in an ice-cold oxygenated sucrose solution ( $3.5 \mathrm{~mm} \mathrm{KCL}, 25 \mathrm{~mm} \mathrm{NaHCO} 3,1.2 \mathrm{~mm} \mathrm{KH}_{2} \mathrm{PO}_{4}$, $1.3 \mathrm{~mm} \mathrm{MgSO}_{4}, 1.2 \mathrm{~mm} \mathrm{CaCl}_{2}, 10 \mathrm{~mm}$ glucose, $212.5 \mathrm{~mm}$ sucrose, and $2 \mathrm{~mm}$ $\left.\mathrm{MgCl}_{2}, \mathrm{pH} 7.4\right)$.

After removing roots and meninges, T13-L3 regions were embedded in low-melting agarose (Sigma-Aldrich) and sectioned transversely at $300-350 \mu \mathrm{m}$ on a vibratome (Vibratome 300 , Vibratome). Slices were incubated in an oxygenated Ringer's solution $(111 \mathrm{~mm} \mathrm{NaCl}, 3.08 \mathrm{~mm}$ $\mathrm{KCl}, 11 \mathrm{~mm}$ glucose, $25 \mathrm{~mm} \mathrm{NaHCO}_{3}, 1.25 \mathrm{~mm} \mathrm{MgSO}_{4}, 2.52 \mathrm{~mm} \mathrm{CaCl}_{2}$, and $1.18 \mathrm{~mm} \mathrm{KH}_{2} \mathrm{PO}_{4}, \mathrm{pH} 7.4$ ) at room temperature for $>30 \mathrm{~min}$ for recovery before recording.

Whole-cell patch-clamp recordings. Following the recovery, slices were transferred to a recording chamber mounted on an Olympus BX51WI microscope and perfused constantly with oxygenated $\left(95 \% \mathrm{O}_{2}+5 \%\right.$ $\mathrm{CO}_{2}$ ) room temperature Ringer's solution. tdTomato fluorescencepositive cells were visually identified using a $40 \times$ water-immersion objective (numerical aperture, 0.8 ) with the aid of a DAGE-MTI IR-1000 CCD camera.

Conventional whole-cell patch-clamp recordings were made in voltage- and current-clamp modes using a MultiClamp 700B amplifier (Molecular Devices). Analog signals were filtered at $10 \mathrm{kHz}$ with the Digidata 1400A board (Molecular Devices) under control of pCLAMP10.3 (Molecular Devices). Patch-clamp recording pipettes with a resistance of 5-8 M $\Omega$ were filled with solution containing $128 \mathrm{~mm}$ K-gluconate, $4 \mathrm{~mm} \mathrm{NaCl}, 0.0001 \mathrm{~mm} \mathrm{CaCl}_{2}, 10 \mathrm{~mm} \mathrm{HEPES}, 1 \mathrm{~mm}$ glucose, $5 \mathrm{~mm} \mathrm{Mg-ATP}$, and $0.3 \mathrm{~mm}$ GTP-Li, pH 7.2. Then $0.4 \mathrm{mg} / \mathrm{ml}$ lucifer yellow dilithium salt (LY; Sigma-Aldrich) and $1 \mathrm{mg} / \mathrm{ml}$ neurobiotin (Vector Laboratories) were added to the pipette solution before recording to facilitate mapping of recorded neurons.

To assure uniform measuring conditions, all cells were held at -60 $\mathrm{mV}$, which is below their action potential threshold, with a tonic DC current. Cells that required $>250 \mathrm{pA}$ of holding current to maintain these potentials or had an action potential amplitude $<60 \mathrm{mV}$ were excluded from the analyzed data. Liquid junction potential was calculated to be $\sim 12 \mathrm{mV}$. The membrane potential values were not corrected.

Fast synaptic currents were blocked by AP-5, NBQX, picrotoxin, and bicuculline in our initial experiments, but we did not observe any significant effects of these antagonists on the cells' intrinsic properties except
Table 1. Electrophysiological properties of V3 spinal INs

\begin{tabular}{|c|c|c|c|}
\hline Electrophysiological properties & Cluster $1(N=65)$ & Cluster $2(N=57)$ & $p$ value \\
\hline Input $R(M \Omega)$ & \multicolumn{3}{|c|}{$233.092 \pm 132.0881038 .522 \pm 648.563<0.001$} \\
\hline$C_{m}(p F)$ & $66.477 \pm 15.770$ & $29.263 \pm 9.768$ & $<0.001$ \\
\hline Resting membrane potential (mV) & $-44.778 \pm 2.576$ & $-47.123 \pm 3.374$ & $<0.001$ \\
\hline$f-I$ slope (average spike frequency) & $0.172 \pm 0.0737$ & $0.827 \pm 0.293$ & $<0.001$ \\
\hline$f-/$ slope (1st spike) & $1.639 \pm 1.686$ & $1.502 \pm 1.127$ & 0.047 \\
\hline $10 \mathrm{HZ}$ 1st spike latency (s) & $0.0189 \pm 0.00992$ & $0.0484 \pm 0.0197$ & $<0.001$ \\
\hline $10 \mathrm{HZ}$ 1st spike frequency (Hz) & $53.195 \pm 34.406$ & $18.878 \pm 14.393$ & $<0.001$ \\
\hline $10 \mathrm{HZ}$ Spike frequency adaptation & $7.525 \pm 6.459$ & $1.437 \pm 0.700$ & $<0.001$ \\
\hline Rheobase (pA) & $38.672 \pm 21.710$ & $8.745 \pm 6.654$ & $<0.001$ \\
\hline 1st spike latency (s) & $0.204 \pm 0.435$ & $0.348 \pm 0.198$ & $<0.001$ \\
\hline 1st spike amplitude (mV) & $93.258 \pm 9.791$ & $87.565 \pm 11.286$ & 0.004 \\
\hline 1st spike absolute amplitude & $37.865 \pm 10.829$ & $28.732 \pm 10.590$ & $<0.001$ \\
\hline 1st spike threshold (mV) & $-41.337 \pm 4.609$ & $-39.763 \pm 4.625$ & n.s. \\
\hline 1st spike width (ms) & $0.969 \pm 0.230$ & $0.915 \pm 0.225$ & n.s. \\
\hline $80 \mathrm{pA}$ spike number & $8.063 \pm 6.060$ & $38.688 \pm 11.126$ & $<0.001$ \\
\hline $10 \mathrm{HZ}$ afterstimulus hyperpolarization & $6.372 \pm 2.661$ & $2.402 \pm 1.173$ & $<0.001$ \\
\hline Sag amplitude at $-120 \mathrm{mV}(\mathrm{mV})$ & $26.137 \pm 6.572$ & $12.865 \pm 4.549$ & $<0.001$ \\
\hline Sag membrane potential slope & $-0.437 \pm 0.132$ & $-0.217 \pm 0.0801$ & $<0.001$ \\
\hline Latency of rebound spike at $-120 \mathrm{mV}$ (s) & 5) $0.0285 \pm 0.0333$ & $0.114 \pm 0.0610$ & $<0.001$ \\
\hline Latency of 1st rebound spike (s) & $0.0845 \pm 0.0524$ & $0.272 \pm 0.341$ & $<0.001$ \\
\hline $\begin{array}{l}\text { Membrane potential of 1st rebound } \\
\text { spike }(\mathrm{mV})\end{array}$ & $-82.541 \pm 9.220$ & $-96.602 \pm 14.987$ & $<0.001$ \\
\hline
\end{tabular}

Data are given as the mean $\pm S D$.

for the membrane input resistance. Therefore, we conducted all our subsequent experiments without any of the blockers. During some recording protocols, action potentials were blocked with $0.1 \mu \mathrm{M}$ TTX (Alomone Labs) added to the extracellular medium. Other drugs used included ZD7288 (10 $\mu \mathrm{M}$; Tocris Bioscience) to block hyperpolarizationactivated cation channels $\left(I_{\mathrm{h}}\right)$ and $\mathrm{NiCl}_{2}(0.15-0.3 \mathrm{~mm}$; Sigma-Aldrich), NNC 55-0396 hydrate (30 $\mu \mathrm{M}$; Sigma-Aldrich), and mibefradil (10 $\mu \mathrm{M}$, Sigma-Aldrich) to block T-type calcium channels (ICaT).

\section{Data acquisition and analysis}

Data were obtained by pClamp 10.3 (Molecular Devices), and analyzed by Clampfit 10.3 and Spike2 5.0 (Cambridge Electronic Design) software packages.

Input resistance and capacitance were evaluated by measuring the cells' response to repetitive, small negative steps $(-10 \mathrm{mV}, 100 \mathrm{~ms})$. To obtain frequency-current $(f-I)$ plots, 1 s current pulses with increments of 5,10 , or $20 \mathrm{pA}$ were applied to the cell. The average spike frequency was determined by counting the number of spikes during the $1 \mathrm{~s}$ pulse. The instantaneous frequency was calculated by measuring the first interspike interval of each step. Spike frequencies were plotted against injected currents. In our experiments, the spike frequency of some small cells quickly reached a plateau stage with the depolarization series. To avoid wrongly fitting the saturation phase, the $f-I$ curve was fitted by the equation $y=a \times x /(1+x / b)$, where $a$ represents the initial linear slope of the $f-I$ plot and $b$ is the presumed maximum spike frequency. The spike frequency adaptation (SFA) ratio was calculated by the average of the last three interspike intervals divided by the average of the first three interspike intervals at the step at which the average spike frequency of the cell was $10 \mathrm{~Hz}$. We also measured the rheobase, spike latency, amplitude, half-width, and threshold of the first spike generated by the smallest suprathreshold current.

Subthreshold properties of cells were obtained by applying $1 \mathrm{~s}$ current pulses to different hyperpolarization membrane potentials. Sag voltage amplitude was defined as the difference between the peak of the hyperpolarized membrane potential and the steady-state voltage.

The 21 attributes that we used in our cluster analyses comparison are listed in Table 1.

\section{Morphological analysis}

After the electrophysiological recordings were completed, slices containing cells marked by LY/neurobiotin were fixed in $4 \%$ paraformaldehyde for $0.5-1 \mathrm{~h}$ and rinsed three times in $0.1 \%$ Triton X-100 (PBS-T). After overnight incubation with streptavidin conjugated with Alexa Fluor 488 
(Invitrogen), the images were obtained using a Zeiss LSM 510 upright confocal microscope and fluorescence microscope (DMLPS, Leica), and cell morphological structures were analyzed. The area of the cell soma was measured using ImageJ. The neural branches were drawn and counted manually by following the fluorescent signals under the fluorescence microscope (DMLPS, Leica). The accuracy of the drawing and counting was confirmed by parallel drawings using the program Neurolucida (MBF Bioscience) under an Olympus BX 50 upright microscope and blinded observers.

Except for the slices used above, all other cross sections were obtained directly from freshly dissected spinal cords at different ages. Embryonic tissue was obtained at embryonic day 10.5 (E10.5) and E14.5. Pregnant female mice were euthanized by cervical dislocation after deep anesthesia via inhaled isoflurane. Embryos were rapidly dissected out of the uterus and fixed whole for E10.5 and isolated spinal cords for E14.5 for $1 \mathrm{~h}$ on ice in $4 \%$ paraformaldehyde in $0.01 \mathrm{M}$ PBS, pH 7.4, cryoprotected in $20 \%$ sucrose in $0.01 \mathrm{M}$ PBS overnight. Postnatal tissue was collected from P0 mice by decapitation, and the spinal cord was isolated, fixed for $1 \mathrm{~h}$ in $4 \%$ paraformaldehyde, and cryoprotected in 20\% sucrose in $0.01 \mathrm{~m}$ PBS overnight. The tissue from the P21 spinal cord was obtained following the procedure described in the later section of the c-fos (a protooncogen) experiment procedures. All tissues were embedded in octreotide (OCT) for cryostat sectioning. The $30-\mu \mathrm{m}$-thick cryostat sections were collected onto slides for immunolabeling. Sections were washed in $0.01 \mathrm{M}$ PBS with $0.1 \%$ Triton X-100 (PBS-T), blocked for $1 \mathrm{~h}$ in $10 \%$ heat-inactivated normal horse serum in PBS-T, and incubated overnight in anti-dsRed primary antibodies (Clontech) in PBS-T at either $4^{\circ} \mathrm{C}$ or room temperature. Donkey secondary antibodies against rabbit conjugated to Cy3 (Jackson ImmunoResearch) were diluted 1:400. Sections were washed in PBS and coverslipped with Vectashield anti-fade mounting medium (Vector Laboratories). The images were obtained using Zeiss Axiovert 200M fluorescent microscope. The dsRed primary antibody can specifically recognize the tdTomato protein. We use it to enhance and preserve the fluorescent signal in V3 INs.

\section{Statistics}

Student's $t$ test for electrophysiological properties and Mann-Whitney $U$ test for morphological properties were used to determine statistical significances between groups. A paired $t$ test was used to assess drug effects (SigmaPlot 11.0, SYSTAT). Significance is indicated as ${ }^{\star} p \leq 0.05$ and ${ }^{* *} p \leq 0.01$. Data in the Results represent the mean \pm SD.

\section{Cluster analysis}

We used a standard cluster algorithm (the clusterdata function in Matlab) to identify subset of the electrophysiological properties (attributes) that exhibited the best separation into distinct clusters. The criterion used to identify best separation consisted of the product of the ratio of the between-cluster variance to the total variance (denoted $h^{2}$ ), and the relative diversity index (rdi). $h^{2} \times$ rdi is a number between 0 and 1 , such that values closer to 1 indicate good separation between clusters $\left(h^{2} \approx 1\right.$; Ma et al., 2006) that are approximately equal in size $(\mathrm{rdi} \approx 1)$.

For a given set of attributes, the data were compiled into a matrix, $M$, with a row for each neuron and a column for each attribute. Neurons were then assigned to one of a predefined number of clusters using the cluster algorithm. The between-cluster variance was then computed as follows. $M$ was first centered by subtracting the mean vector to give $M_{c}=$ $M-\bar{M}$. Each column of $M_{c}$ was divided by its SD to account for differences in the dynamic ranges of the attributes. The matrix $W$ of intracluster differences was then computed by subtracting from each row of $M_{c}$ the mean of the cluster that it was assigned to. The result was used to compute the matrix of intercluster differences, $B=W-M_{c}$. The ratio of the intercluster variance to the total variance was then calculated using the equation $\eta^{2}=$ variance $(B) /$ variance $\left(M_{c}\right)$.

The rdi was computed from the assigned clusters as follows. Given $k$ clusters for which the proportion of observations in each is $p_{j}$, the Gini index (Hastie et al., 2009) is $I_{\text {Gini }}=1-\sum P_{i}^{2}$. The Gini index attains its maximum value when the data are equally distributed between clusters, in which case all of the $p_{j}$ values are equal to $1 / k$. We divided the Gini index by its maximum value to produce an index between 0 and 1 , namely rdi $=I_{\text {Gini }} /\left(1-\Sigma k^{-2}\right)$.
Combinations of attributes for which the largest criterion value was $>0.90$ were considered for further analysis, which consisted of visual inspection of the histogram of the first principal component (PC1) of $M$ for evidence of a multimodal distribution. A criterion of parsimony was also applied, meaning that combinations of fewer attributes were deemed to be superior compared with those consisting of more attributes, all else being equal.

\section{c-Fos induction with different locomotor activities}

Young adult Sim $1^{\mathrm{Cre} /+}$; Rosa $a^{\text {floxstop26TdTom }}$ mice (P32-P35) were randomly placed in the following three groups: control $(n=3)$, running $(n=3)$, and swimming $(n=3)$. Running or swimming groups were kept on a treadmill at a speed of $0.15-0.20 \mathrm{~m} / \mathrm{s}$ for $3 \times 20 \mathrm{~min}$ or in a water tank for $4 \times 10 \mathrm{~min}$, respectively. During swimming, two 0.5 -cm-wide plastic loops were placed under the sternum of mice to prevent drowning. Control mice were kept in the home cage in a quiet place for $60 \mathrm{~min}$. Mice were then anesthetized by intraperitoneal injection of a mixture of ketamine and xylazine, and transcardially perfused with $0.1 \mathrm{M}$ PBS, followed by $4 \%$ paraformaldehyde. Spinal cords were harvested and postfixed in $4 \%$ paraformaldehyde at $4^{\circ} \mathrm{C}$ overnight and then cryoprotected in $20 \%$ sucrose before embedding in OCT and cryostat sectioning. The $30-\mu \mathrm{m}$-thick sections were collected for immunolabeling. Sections were washed in $0.01 \mathrm{M}$ PBS with $0.1 \%$ Triton X-100 (PBS-T), blocked for $1 \mathrm{~h}$ in $5 \%$ normal goat serum in PBS-T, and incubated at room temperature overnight in rabbit anti-Fos antibody (1:300; Santa Cruz Biotechnology) in PBS-T with $2 \%$ goat serum. Sections were washed in $3 \times$ PBS-T and incubated with goat anti-rabbit conjugated to Alexa Fluor 488 (1:500; The Jackson Laboratory) for $2 \mathrm{~h}$ at room temperature. Sections were then washed in PBS three times and coverslipped with anti-fade mounting medium (Dako). The images were obtained using a Zeiss LSM 510 upright confocal microscope and a Zeiss Axiovert 200M fluorescent microscope. Total Tdtomato-positive V3 neurons and those coexpressing Fos protein were counted and mapped. For each animal, we sampled a total of 20 sections from the lumbar region, and summarized the relative position of all double-labeled cells onto one schematic cross section. In addition to using the central canal and Rexed's laminae as landmarks, we also set grids on the image of transverse sections of the spinal cord and the schematic section to more accurately map the double-labeled cells.

The proportion of activated V3 neurons (c-Fos-positive cells) was calculated for each of the nine combinations of the two factors: neuron location (ventral, intermediate, and dorsal), and activity undertaken (control, running, swimming). For each factor combination, the proportions for the three mice $\left(p_{1}, p_{2}, p_{3}\right)$ were averaged to produce a single estimate $p_{\text {avg }}=1 / 3 \sum_{i=1}^{3} p_{i}$. Note that the SE for each proportion $p_{i}$ is given by $\mathrm{sd}_{i}=\sqrt{p_{i}\left(1-p_{i}\right) / n_{i}}$, and the SE for the average $\left(p_{\text {avg }}\right)$ by $\operatorname{sd}_{\text {avg }}=1 / 3 \sqrt{\sum_{i=1}^{3} \mathrm{sd}_{i}^{2}}$. Comparisons of $p_{\text {avg }}$ for each location across activities were made using a two-tailed $z$-test at a level of significance of 0.01 .

\section{Results \\ V3 spinal interneurons display distinctive distribution patterns in the spinal cord}

V3 neurons are marked by the transient expression of the Sim1 transcription factor and arise from the p3 progenitor domain (Zhang et al., 2008). For all the experiments described here, we used a tdTomato fluorescence reporter (Madisen et al., 2010) that is activated by a Sim1Cre knock-in driver (Zhang et al., 2008) to fluorescently mark the V3 IN population in the embryonic and postnatal spinal cord. Between E10 and E11, postmitotic V3 INs that express Sim 1 were identified immediately above the floor plate (Fig. 1A; Zhang et al., 2008). They then migrate dorsally and laterally during postmitotic development (Fig. 1B). By birth, a major group of ventral commissural V3 INs were seen to be present in lamina VIII of the ventral horn (Fig. 1; Zhang et al., 2008). Sim1 V3 INs were also present in laminae VI, VII, and X (the intermediate zone), as well as in laminae IV-V of the spinal cord (Fig. 1C; Geiman et al., 2006). These clusters of V3 INs in both the 
ventral spinal cord and the deep dorsal horn were seen to persist until at least P35 (Fig. 1D; see Fig. 8, P35). Overall, these observations indicate that V3 INs that are born in the $\mathrm{p} 3$ progenitor domain, which is adjacent to the floorplate, become widely distributed in the spinal cord.

Such distribution, however, was not identical at different segments along the spinal cord. The dorsal clusters of V3 INs were only observed in the thoracic and upper lumbar segments (Fig. 1Ei, ii). At the lower lumbar and sacral segments, V3 INs were only located in the ventral side of the spinal cord (Fig. 1Eiii, iv), and similar distribution was also seen in cervical region (data not shown). The molecular mechanism underlying such segmental arrangement is unknown. In our current study, however, we will only focus on the most broadly distributed V3 INs located in the lower thoracic and upper lumbar segments, which are supposedly important for hindlimb movements.

\section{V3 spinal interneurons are an electrophysiologically heterogeneous population}

We ask here whether broadly distributed V3 INs display any diversity in their intrinsic properties that would indicate the presence of subpopulations with distinct physiological, and thus potentially functional, properties. To examine the electrophysiological properties of V3 INs, we used whole-cell patch-clamp recordings under voltage-clamp and current-clamp configurations to measure the membrane properties of V3 INs in transverse sections from the T13-L3 spinal cord region of Sim1 $1^{\mathrm{Cre} /+} ; R 26^{\text {floxstop-tdTom }}$ mice at ages P21P23. Neurobiotin and LY were included in the intracellular buffer to identify the recorded cells post hoc and to assess their morphology (Fig. 2Ai, Bi). Under current-clamp conditions, most V3 INs manifested spontaneous activity without injected current (data not shown). Therefore, we held the membrane potential of all cells at $-60 \mathrm{mV}$ to eliminate spontaneous activity and make initial conditions uniform when measuring their responses to injected currents (Fig. 2).

We first characterized the spike patterns of $\mathrm{V} 3 \mathrm{IN}$ in in response to suprathreshold current steps. We found that all recorded V3 INs generated continuous repetitive spikes (Fig. 2A,B). More detailed analysis, however, revealed that firing properties across the entire V3 population showed broad variation. For example, although all V3 INs can generate repetitive spikes, approximately half showed a tonic or slightly adaptive firing pattern (Fig. $2 B, C$ ), while others had strong spike frequency adaptation, and $\sim 30 \%$ of adaptive cells showed clear initial bursts (Fig. 2A,C). The instantaneous spike frequency (first spike frequency) of some strongly adapting cells reached $>100 \mathrm{~Hz}$, but then decreased quickly when the depolarization pulse continued (Fig. 2C). When we used the SFA ratio at $10 \mathrm{~Hz}$ as an adaptation indicator, adaptation values ranged from 0.98 to 23 . Another parameter that varied considerably was the gain of spikes in response to suprath-
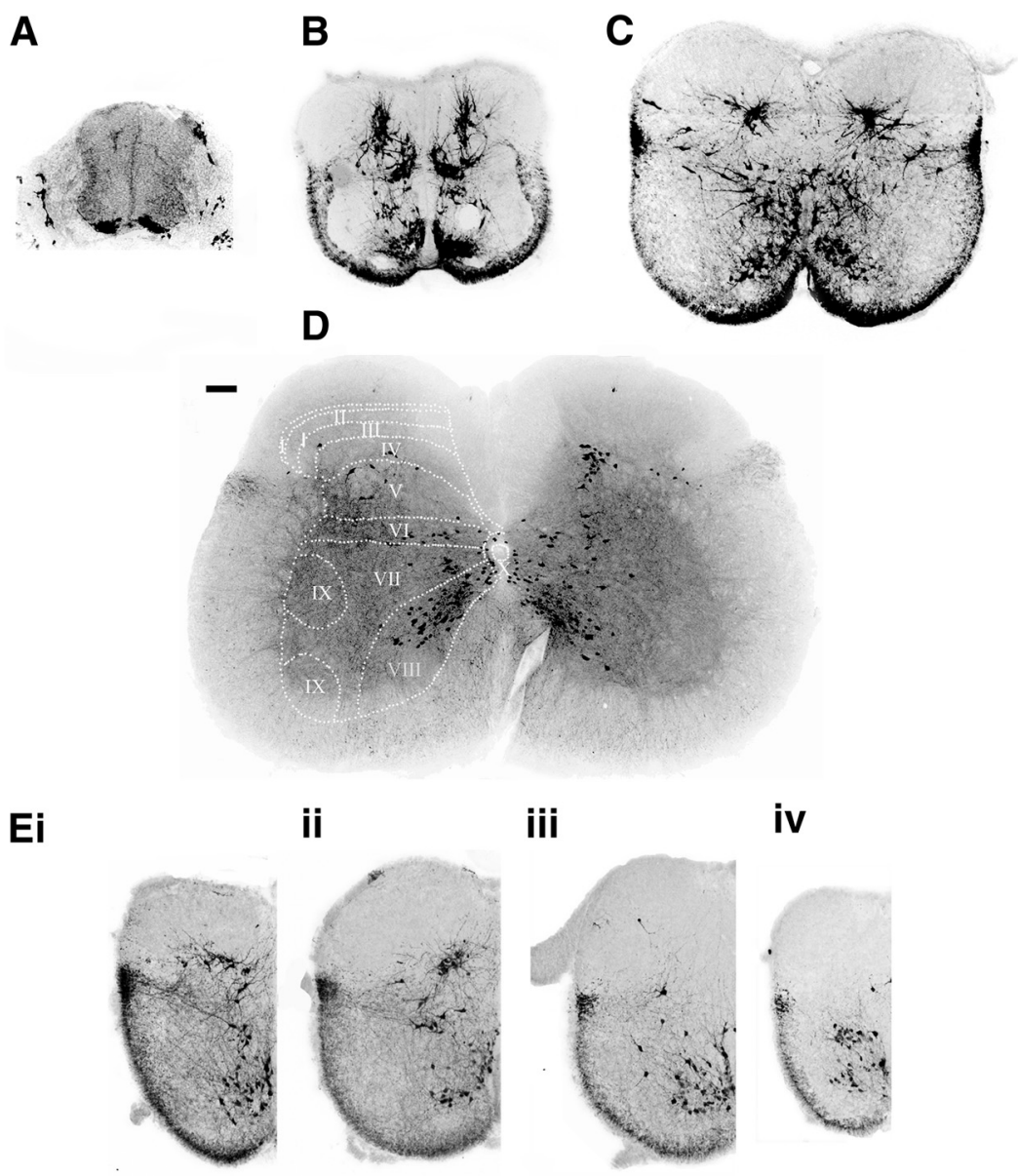

iv

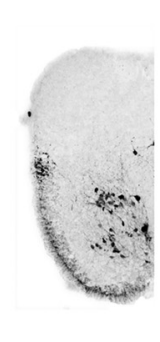

Figure 1. Distribution patterns of $\mathrm{V} 3 \mathrm{INs}$ in the mouse spinal cord. $\boldsymbol{A}-\boldsymbol{D}$, The $30 \mu \mathrm{m}$ transverse sections from lumbar segments of spinal cords from Sim1 ${ }^{\text {Cre/+ }}$; Rosa ${ }^{\text {floxstop26TdTom }}$ mice, at E10.5 (A), E14.5 (B), P0 (C), and P21 (D). Scale bar, $100 \mu \mathrm{m}$. The dashed lines and numbers indicate the Rexed's laminae. $\boldsymbol{E}$, The $30 \mu \mathrm{m}$ half-transverse sections from a P0 spinal cord at thoracic (i), upper lumbar (ii), lower lumbar (iii), and sacral (iv) regions.

reshold steps, which was indicated by the slope of the frequencycurrent plot (Fig. 2D). Among V3 neurons, it ranged from 0.1 to 1.6 (Fig. 2Giii). It was also evident that most adaptive cells had larger after-stimulus hyperpolarization potentials (s-AHPs; Fig. $2 \mathrm{Aii}$, iii, arrows). Other intrinsic properties, such as input resistance $(830.2 \pm 441.5 \mathrm{M} \Omega)$ and capacitance $(49 \pm 19$ pF; Fig. $2 \mathrm{Gi})$, also showed a large range of variation among cells. Most of these parameters did not exhibit a Gaussian distribution (Fig. 2G), further suggesting that V3 INs are not a homogeneous population. Although we observed a heterogeneous combination of tonic and adaptive spike patterns with V3 INs, we did not observe any delayed, oscillatory, or accelerated patterns, as have been reported by others (Wilson et al., 2005; Smith and Perrier, 2006; Dougherty and Kiehn, 2010; Zhong et al., 2010).

The heterogeneity of V3 INs was also seen in cells' subthreshold properties. When injected with a series of $1 \mathrm{~s}$ pulses to hyperpolarize membrane potential from $-60 \mathrm{mV}$ to different levels, most V3 INs displayed sag voltages and post-inhibitory rebound potentials, but the sizes and kinetics differed significantly (Fig. $2 E)$. Some cells had very small sag voltage, even when hyperpolarized to $-100 \mathrm{mV}$ (Fig. 2Eii). By contrast, others showed large sag voltage even at small hyperpolarization, which increased significantly as hyperpolarization continued (Fig. 2Ei). Similar to firing properties, sag voltage values did not show a Gaussian distribution (Fig. 2Gii). Post-inhibition rebound reactions also var- 

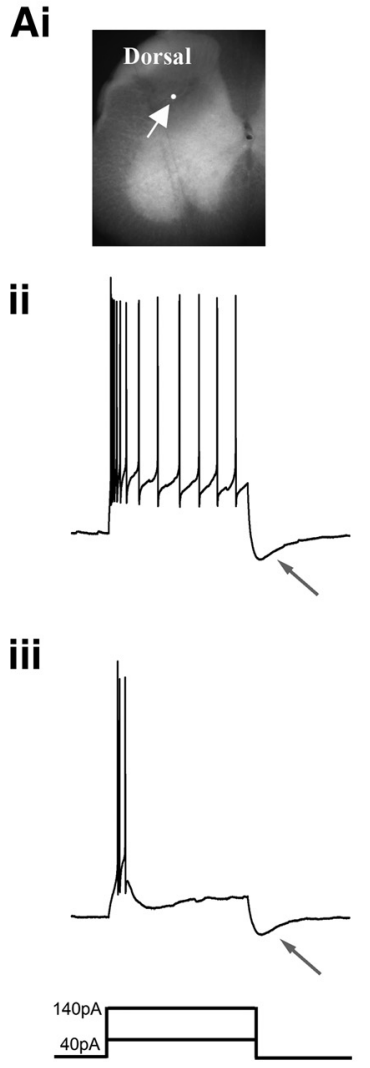

Bi

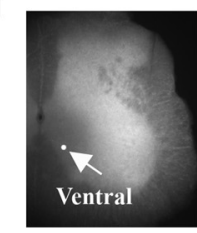

ii

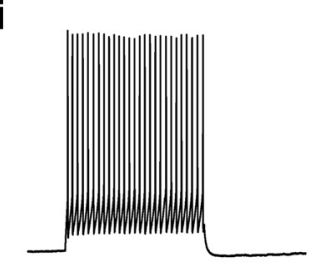

iii

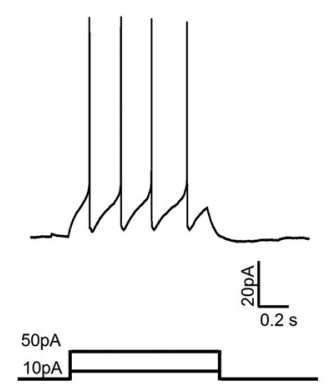

C

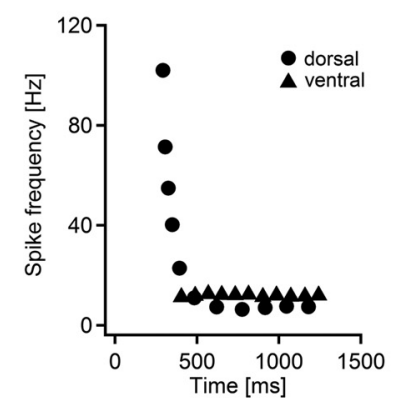

D

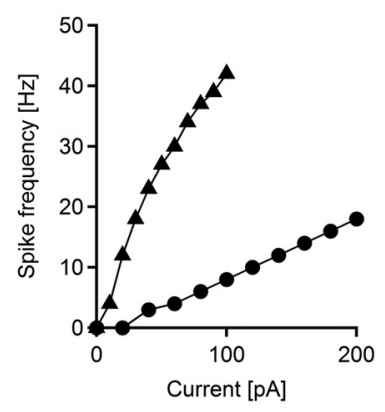

F

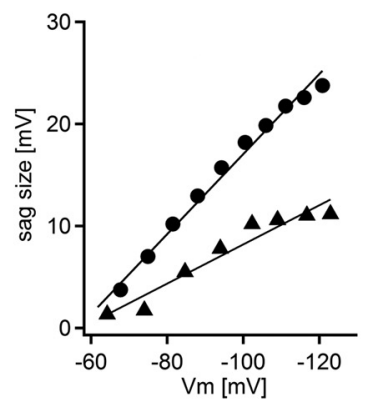

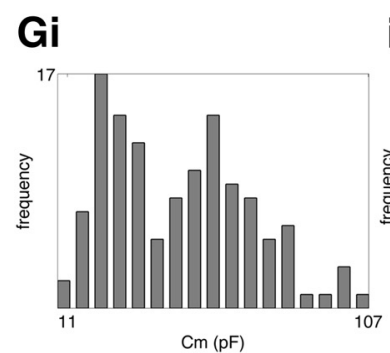

ii

iii

iv
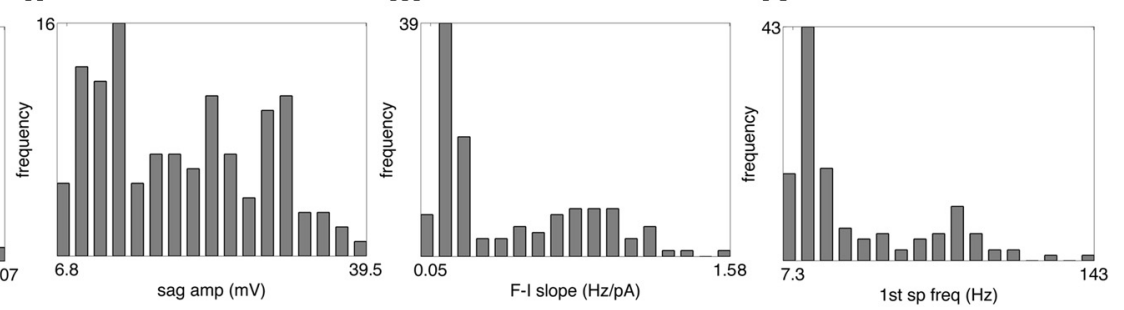

Figure 2. Heterogeneous electrophysiological properties of spinal V3 interneurons. $A, B$, representative traces from two V3 INs responding to $1 \mathrm{~s}$ suprathreshold current steps. $A$, A dorsal V3 IN labeled by neurobiotin $(\boldsymbol{A i})$ displays initial bursts at low (Aiii, $40 \mathrm{pA})$ and high (Aii, $140 \mathrm{pA})$ injected currents. B, A ventral V3 IN (Bi) maintains tonic firing with $10 \mathrm{pA}(\boldsymbol{B i i i})$ and $50 \mathrm{pA}(\boldsymbol{B i i})$ injected currents. White arrows in $\boldsymbol{A i}$ and $\boldsymbol{B i}$ point to the recorded cells; neuron's bodies are labeled with a white dot to show their position. Black arrows in Aii and iii point to the afterhyperpolarization potential after the depolarization pulses. The schematic diagrams show the injected currents. $C$, individual spike frequency is plotted versus time during 1 s injected current on dorsal cell (circles) and ventral cell (triangles). D, Average spike frequency of dorsal cells (circles) and ventral cells (triangles) was plotted versus injected currents. $\boldsymbol{E}$, Representative traces from two V3 neurons hyperpolarized to $-75 \mathrm{mV}$ (black) and $-105 \mathrm{mV}$ (gray). The dashed lines indicate the membrane potentials. $F$, Plots of the size of sag voltage versus membrane potentials of the two cells. $\boldsymbol{G}$, Histograms showing the distribution frequency of various electrophysiological properties of all recorded V3 neurons: $C_{\mathrm{m}}(i)$, sag voltage amplitude (iii), slope of $f-I$ plot (iii), and frequency of first spike (first sp freq, iv).

ied greatly among cells. Some cells exhibited large rebound potentials with a burst of spikes even at relatively low hyperpolarization (Fig. 2Ei). By contrast, other V3 INs generated only small rebound potentials with a few or no spikes at various hyperpolarized membrane potentials (Fig. $2 F$ ). These results further demonstrate that the electrophysiological properties of $\mathrm{V} 3$ INs are not uniform.
In the course of our experiments, we observed that although many suprathreshold and subthreshold properties varied by a large range among spinal V3 INs, cells located in the same "cluster" tended to exhibit similar firing patterns and intrinsic properties. For example, for V3 INs that exhibited a slow $f-I$ slope, strong spike adaptation, and large sag voltage were often found in the deep dorsal horn (Fig. $2 A, C, D, E i$ ), while ventral V3 INs 
tended to have rapid and tonic spikes with small sag voltages (Fig. $2 B-D$,Eii). Therefore, we mapped recorded V3 INs onto a cross section of the spinal cord, and divided them into ventral, intermediate, and dorsal subgroups according to position (Fig. 3A). We then plotted the most commonly observed suprathreshold or subthreshold properties, namely $f-I$ slope, first spike frequency, and sag voltage, of individual neurons in a 3D scheme (Fig. $3 B$ ). This plot illustrated that dorsally located V3 cells were electrophysiologically distinct from those located ventrally, while cells from the intermediate region could display the properties of either (Fig. $3 B$ ). This analysis implied that dorsal and ventral V3 represent distinctive subpopulations with diverse properties, but it was not clear which position the intermediate cells might occupy in the grouping. To quantify the difference in electrophysio-

logical properties between the groups and define the criteria that uniquely identify each, we conducted a systematic cluster analysis.

\section{Cluster analysis identifying V3 subpopulations}

We used a cluster algorithm to assess the 21 attributes of 122 recorded V3 cells (Table 1). We found that a combination of four attributes, $f-I$ slope, membrane capacitance $\left(C_{\mathrm{m}}\right)$, first spike frequency at $10 \mathrm{~Hz}$, and sag amplitude, produced the largest criterion value $\left(h^{2} \times \operatorname{rdi}=0.95\right.$; data not shown). Furthermore, the criterion that allowed for combinations of more than four attributes did not exceed 0.97 . The four-attribute combination was therefore selected based on the criterion of parsimony. The first principle component $\mathrm{PC} 1$ for our selected combination captured $66.5 \%$ of the variance in the data, and its corresponding eigenvector consisted of the $f-I$ slope moving out of phase with the other three attributes, as shown in Figure 4A.

The histogram shown in Figure $4 B$ exhibits two well defined modes, corresponding to positive and negative $\mathrm{PC} 1$ values. This pattern suggested two clusters of V3 neurons according to the sign of PC1, as indicated by light-gray and dark-gray shading. PC1 values $>0$ (cluster 2 ) indicate cells for which the $f-I$ slope is above its mean value and the other three attributes are below their means, while values $<0$ (cluster 1 ) indicate the opposite.

Figure $4 \mathrm{Ci}-i v$ depicts distribution histograms for each of the four attributes. The histogram for the $f-I$ slope attribute (Fig. $4 \mathrm{Ci}$ ) demonstrates good separation between two unimodal distributions, one for each cluster, as does the histogram for the $C_{\mathrm{m}}$ attribute (Fig. 4Cii). Histograms of the remaining attributes, sag amplitude $-120 \mathrm{mV}$ (Fig. 4Ciii) and instantaneous spike frequency (Fig. 4Civ), are more complex: in both, distributions overlap, and it is unclear by inspection whether they are unimodal. Nevertheless, a $t$ test indicated significant separation of cluster means for all four attributes (Table 1). Comparing the distributions, it is noteworthy that the variance appears to increase with the mean of the $f-I$ slope and instantaneous spike frequency (Fig 4Ci,iii). Interestingly, the instantaneous frequency in cluster 1 is more diverse, but $f-I$ slopes vary more in cluster 2 , which may correlate to different functional separations between these two groups of neurons.

When mapped onto a schematic of a spinal cross section, clustered V3 neurons exhibited spatial coherence, as shown in
B

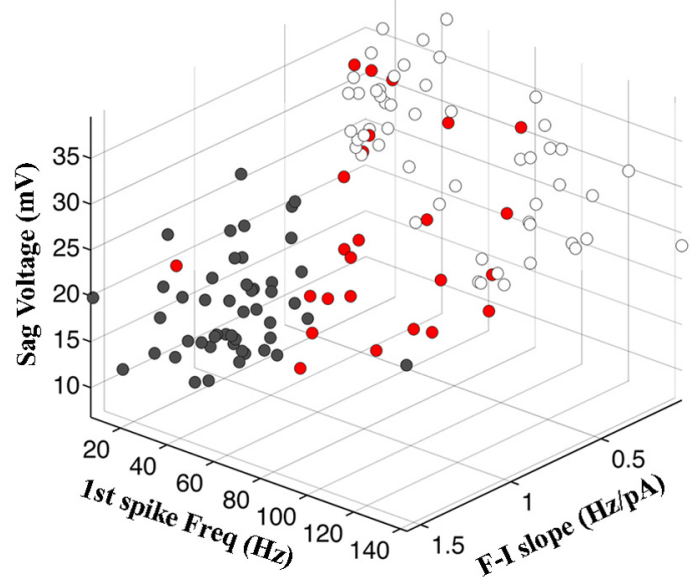

V3 neurons. $A$, All recorded V3 INs were mapped on a half-cross section of spinal cord, and divided into ventral (dark gray), intermediate (red) and dorsal (white) groups). $\boldsymbol{B}$, The slope of the $f-I$ plot was plotted versus the first spike frequency and sag voltage amplitude.

Figure $5 A$. The line marked by a center " $x$ " indicates the principal axis of the location of neurons on the cross section. Plotting the $\mathrm{PC} 1$ value as a function of distance along this axis reveals clearly that the identified clusters are spatially separated, corresponding to the dorsal and ventral regions of the spinal cross section, although the intermediate region contains a mix of both (Fig. 5). This result suggests that V3 INs consist of at least two distinct subtypes based on location. The absence of a gradual transition in the PC1 value between -100 and +100 units along the principal axis (Fig. $5 B$ ) indicates that, although both subtypes exist in the intermediate region, their physiological properties do not change gradually from one subtype to the other, but remain consistent with their respective counterparts in the group. Therefore, we henceforth refer to the two identified clusters as dorsal and ventral subtypes, corresponding to cluster 1 and 2, respectively.

We emphasize that, although the four attributes are the primary factors that separate these clusters, most other electrophysiological properties also differed significantly between these groups (Table 1). Combining all these properties together, we are able to classify our two subgroups of V3 INs: the dorsal V3 INs are low gain and adaptive with large afterhyperpolarization potential, large sag voltage, and large post-inhibitory rebound potential; while ventral V3 INs are high gain and tonic with small afterhyperpolarization potential, sag voltage, and post-inhibitory rebound potential. We next investigated factors that might contribute to the different properties of these two subgroups of V3 INs.

\section{Dorsal and ventral V3 spinal interneurons exhibit unique morphologies}

The clear differences in membrane capacitance values have already suggested that the surface size differs between dorsal and ventral V3 cells. As noted, we included neurobiotin and LY in the intracellular recording buffer, enabling us to visualize the structures of the recorded cells. Slice preparations do not allow us to follow the full length of axons or dendrites and make it difficult to distinguish between them. Thus, we examined the overall architecture of neurons' primary processes within the visual plane. Figure 6, $A i$ and $B i$, shows confocal images of two representative cells from dorsal and ventral clusters, respectively. The former showed greater spatial arborization, while the cell body of the latter and its primary branches lay along a horizontal plane. We 
A
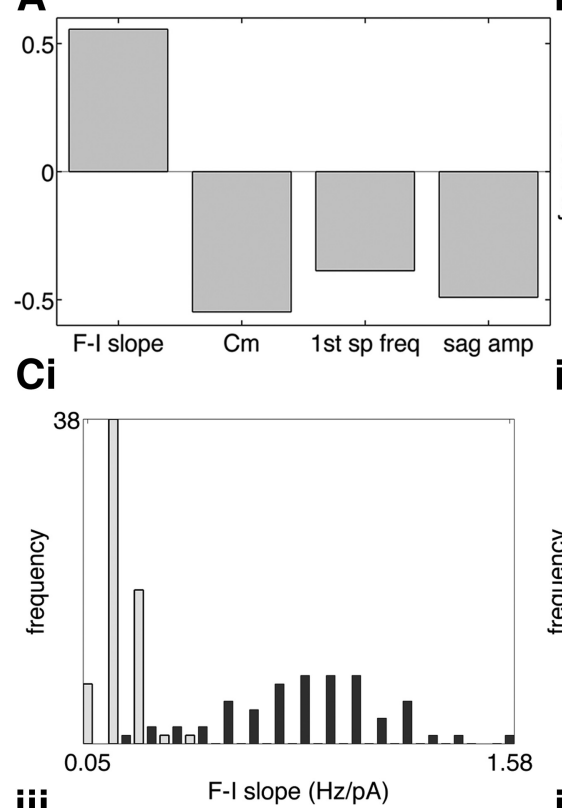

iii

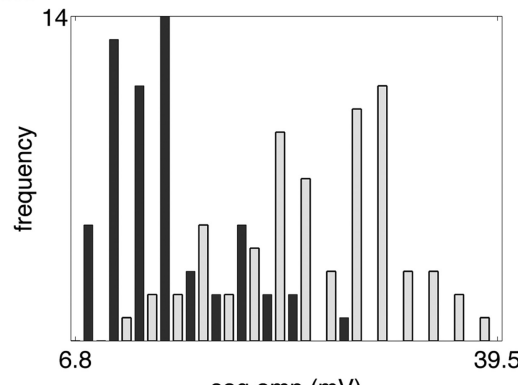

sag amp (mV)
B
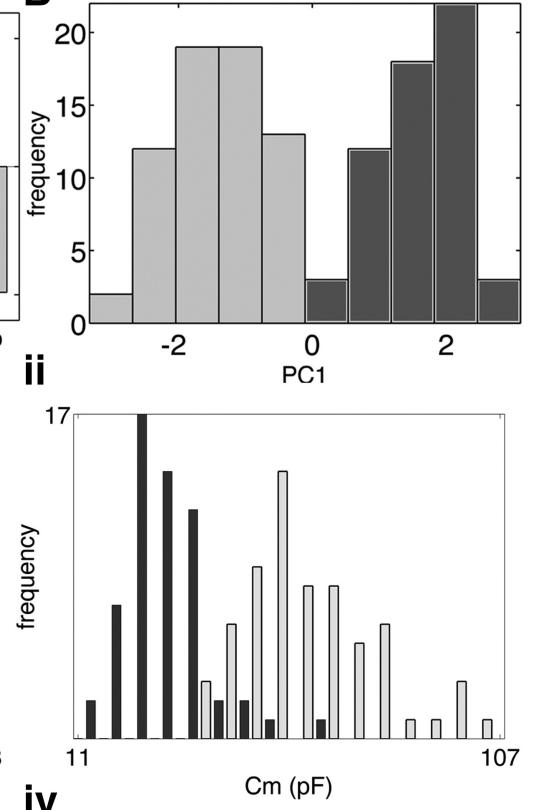

iv

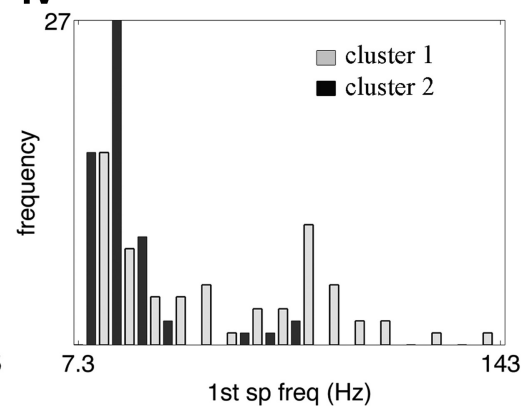

Figure 4. Cluster analysis of $\mathrm{V} 3$ neurons based on electrophysiological properties. $\boldsymbol{A}$, The first eigenvector of four attributes ( $f-I$ slope, $C_{m}$, first spike frequency, and sag amplitude at $\left.-120 \mathrm{mV}\right)$ that produced the best criterion. $\boldsymbol{B}$, Histogram of $\mathrm{PC} 1$ exhibits two well defined modes corresponding to positive and negative values representing cluster 1 (PC1 $<0$, light gray) and cluster 2 (PC1 $>0$, dark gray). $\boldsymbol{C}-\boldsymbol{i} \boldsymbol{i}$, Histogram of distribution of the four main attributes $(\boldsymbol{A})$ with cells in cluster 1 (light gray) and cluster 2 (dark gray).

A

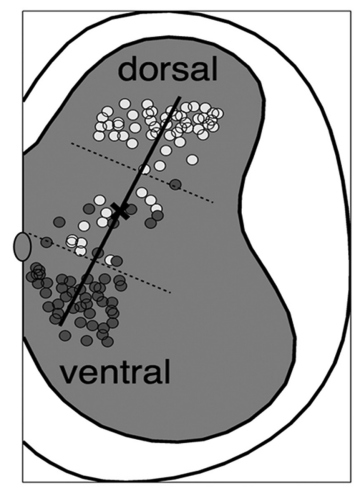

B

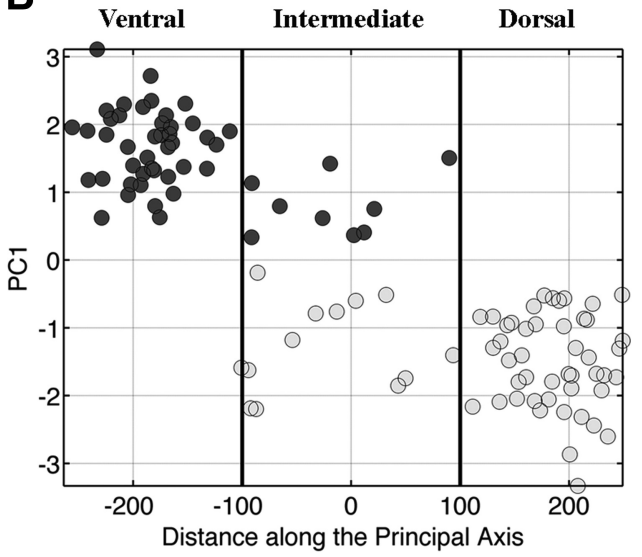

Figure 5. Electrophysiological clusters of V3 INs are topologically distributed in the spinal cord. $A$, Cross-section map of V3 neurons divided into cluster 1 (light gray) and cluster 2 (dark gray). The dark line indicates the principal axis of the

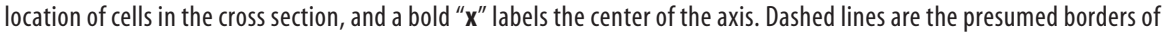
ventral, intermediate, and dorsal separations. $\boldsymbol{B}$, Plot of the value of PC1 as a function of distance along the axis. The dashed lines in $\boldsymbol{A}$ and the dark lines in $\boldsymbol{B}$ indicate the arbitrary borders to separate the ventral, intermediate, and dorsal populations.

further recovered and analyzed 67 neurons (Fig. 6Aii,Bii; Table 2). In general, V3 INs from the dorsal cluster exhibited larger somas and a more complex morphology than did ventral cells (Fig. 6; Table 2); ventral V3 INs had fewer initial processes, and their processes were less branched than dorsal cells, while initial processes of dorsal cells exhibited complicated branching immediately adjacent to the soma. The number of both initial branches and total branches in our planes of view was greater in dorsal compared with ventral cells (Table 2). The distribution frequencies of values representing soma area and the number of branches also clearly indicated distinct ventral and dorsal subpopulations (Fig. 6C; Table 2). Such morphological and structural differences can also be attributed to the diversity of electrophysiological properties between V3 neurons.

\section{Ion channel currents shape the electrophysiological properties of V3 spinal interneurons}

Observed differences in both the intrinsic and firing properties of ventral and dorsal V3 neurons strongly suggest that they express a different compliment of ion channels. First, we assessed the effects of ZD7288, a specific $I_{\mathrm{h}}$ blocker, on recordings from V3 INs. ZD7288 substantially blocked sag voltage in all V3 INs (Fig. $7 A, B$, dark gray), but in some cells a small portion of a slow component of sag voltage in dorsal V3 INs remained (Fig. 7Ai, dark gray). This result indicates V3 INs in both group express $I_{\mathrm{h}}$ channels. ZD7288 treatment increased the input resistance by $189.7 \pm 100 \%(n=9)$ in dorsal cells compared with $52.5 \pm 20.7 \%(n=12)$ in ventral cells $(p=0.002)$ and hyperpolarized membrane potentials of all V3 INs, but by $-12.1 \pm 4.1 \mathrm{mV}$ in dorsal cells compared with $-5.9 \pm 3.6 \mathrm{mV}$ in ventral cells $(p<0.05)$. A more significant difference between the two populations was the response of their rebound potentials. ZD7288 treatment negated almost all rebound potentials and spikes in ventral V3 INs but only slightly reduced rebound potentials in dorsal cells, even though the latency of their first spike was increased (Fig. 7A, dark gray). Additional treatment with $0.3 \mathrm{~mm}$ nickel (Fig. $7 A i$, light gray), which blocks ICaTs eliminated rebound potentials and spikes of dorsal cells completely $(n=9)$. To further confirm the potential involvement of ICaT in dorsal V3 INs, we used two other selective blockers, NNC 55-0396 (NNC; Huang et al., 2004) and mibefradil (McDonough and 
Bean, 1998). In all cases ( $n=5$ for NNC; $n=2$ for mibefradil), the rebound potential and spikes of dorsal cells were completely removed by these two blockers (Fig. 7Aii). These results further indicate that ICaT may account for the large rebound response in the dorsal V3 INs. We also noticed a small residual sag voltage that could be seen in some dorsal V3 INs after ZD7288 application could also be flattened by nickel (Fig. 7Ai), which may imply more complex ion channel components playing roles in dorsal V3 INs.

We also examined the effects of ZD7288 and nickel on the firing properties of V3 subpopulations (Fig. 7C-F). Overall, treatment with ZD7288, but particularly with both blockers simultaneously, had significant effects on the spike rate and adaptation ratio of dorsal cells (Fig. 7C,E). For example, ZD7288 treatment alone increased the slope of the $f-I$ plot of dorsal cells from $0.179 \pm 0.033$ to $0.263 \pm 0.0906(n=9, p=0.03)$ and increased the firing frequency in response to injected currents at all levels (Fig. 7E). Following the addition of nickel, increases in both slope and firing frequency were further intensified (Fig. $7 C, E$ ). The $f-I$ plot slope increased to $0.346 \pm 0.143(p=$ 0.014; Fig. 7E). ZD7288 together with nickel also reduced the first spike frequency of the dorsal cells (control, $57.0 \pm$ 34.4 vs $44.6 \pm 21.3 \mathrm{~Hz} ; p=0.005$ ). The same effects could also be observed with NNC and mibefradil (data not shown). However, neither blocker had significant effects on the firing properties of ventral group cells except on the firing frequency with 40 and $60 \mathrm{pA}$ injected currents (Fig. $7 D, F)$. Furthermore, ZD7288 with nickel also eliminated the s-AHP in the dorsal cells (Fig. 7C). Overall, these results suggest that $I_{\mathrm{h}}$ and ICaT currents may potentially play more significant roles in shaping the properties of dorsal rather than ventral V3 neurons.

\section{Dorsal and ventral V3 INs are differentially recruited for distinct locomotor functions}

To investigate the functional differences between these subpopulations, we subjected young adult (P32-P35) Sim1 ${ }^{\mathrm{Cre} /+}$; Rosafloxstop26TdTom mice to two distinct locomotor tests and assessed neuronal activity by c-Fos expression (Dai et al., 2005; Fig. 8A). We randomly assigned three animals in each group. One group was placed on a treadmill and ran at the rate of 20 $\mathrm{cm} / \mathrm{s}$ for $60 \mathrm{~min}(3 \times 20 \mathrm{~min})$, while the other was made to swim for $\sim 40 \mathrm{~min}(4 \times 10 \mathrm{~min})$. Control animals remained in home cage for $60 \mathrm{~min}$. Forty to $60 \mathrm{~min}$ after the tasks, T13-L4 spinal cords of the tested animals were isolated; V3 neurons with and without c-Fos expression were counted and mapped in spinal cord cross sections (Fig. 8B); and histograms were constructed to show the proportion of c-Fos-positive cells ver-

\section{ii}

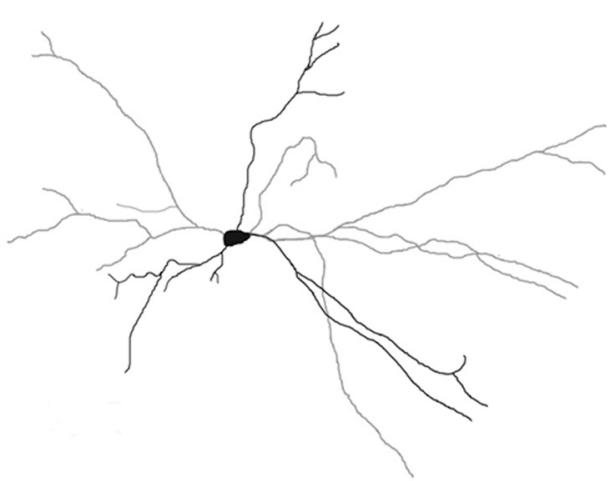

ii

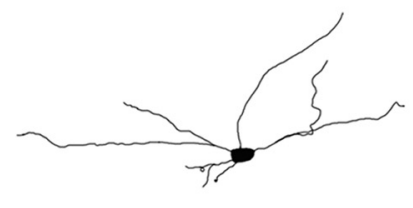

ii

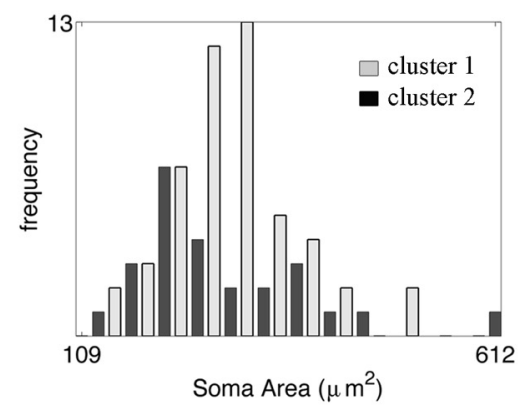

Figure 6. The morphological properties of dorsal and ventral V3 clusters. Ai, Bi, Confocal images of representative V3 INs from usters 1 (Ai) and 2 (Bi). Colors reflect the depth of the image from the surface (red) to deep into the section (blue). Aii, Bii, Reconstruction of corresponding cells in $\mathbf{A i}$ and $\mathbf{B i}$. Ci, Distribution histogram of primary branches of cells in clusters 1 (light gray) and 2 (dark gray). Cii, Distribution histogram of soma areas of cells in clusters 1 (light gray) and 2 (dark gray).

Table 2. Morphological properties of V3 spinal interneurons

\begin{tabular}{lccc}
\hline Morphological properties & Cluster $1(N=44)$ & Cluster $2(N=23)$ & $p$ value \\
\hline Body area $(\mu \mathrm{m})$ & $301.782 \pm 82.153$ & $261.804 \pm 109.803$ & 0.018 \\
Initial branches number & $5.25 \pm 1.314$ & $4.522 \pm 1.238$ & 0.027 \\
Total branches number & $13.182 \pm 4.133$ & $9.826 \pm 4.697$ & 0.002 \\
Branches index & $2.573 \pm 0.787$ & $2.124 \pm 0.722$ & 0.03 \\
\hline
\end{tabular}

Data are given as the mean \pm SD.

sus total tdTomato-positive cells at ventral, dorsal, and intermediate positions, and compared their respective $99 \%$ confidence interval between each groups (Fig. 8C). This analysis showed that in control awakened animals, many spinal neurons were c-Fos positive, including an average of $22 \%$ ventral, $11.0 \%$ intermediate, and $9.6 \%$ dorsal V3 INs (Fig. $8 B, C$ ). After running or swimming, the number of activated V3 neurons remarkably increased, and $\sim 50 \%$ of ventral V3 INs and $30 \%$ of intermediate V3 INs became c-Fos positive (Fig. 
$\mathbf{A i}$

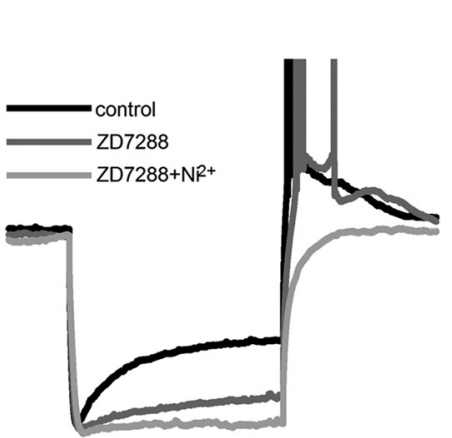

ii

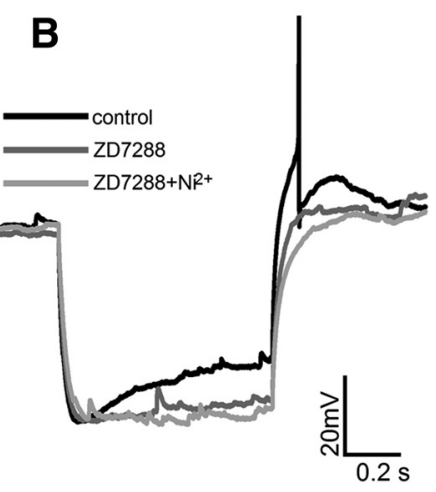

C
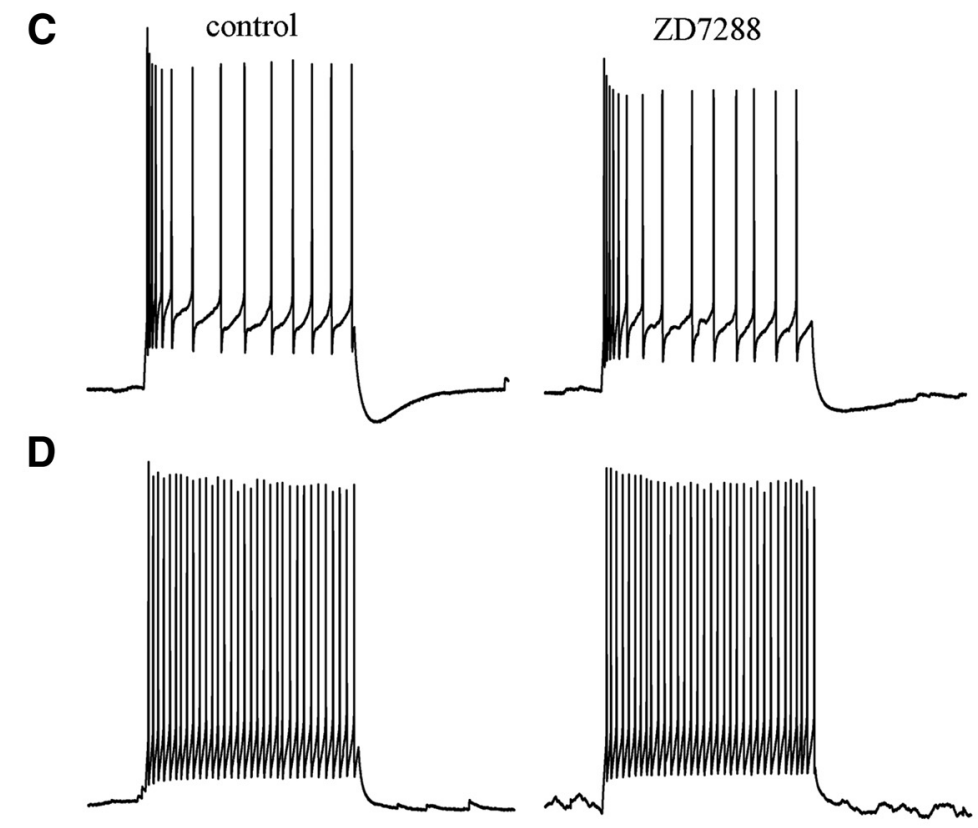

$\mathrm{ZD} 7288+\mathrm{Ni}^{2+}$
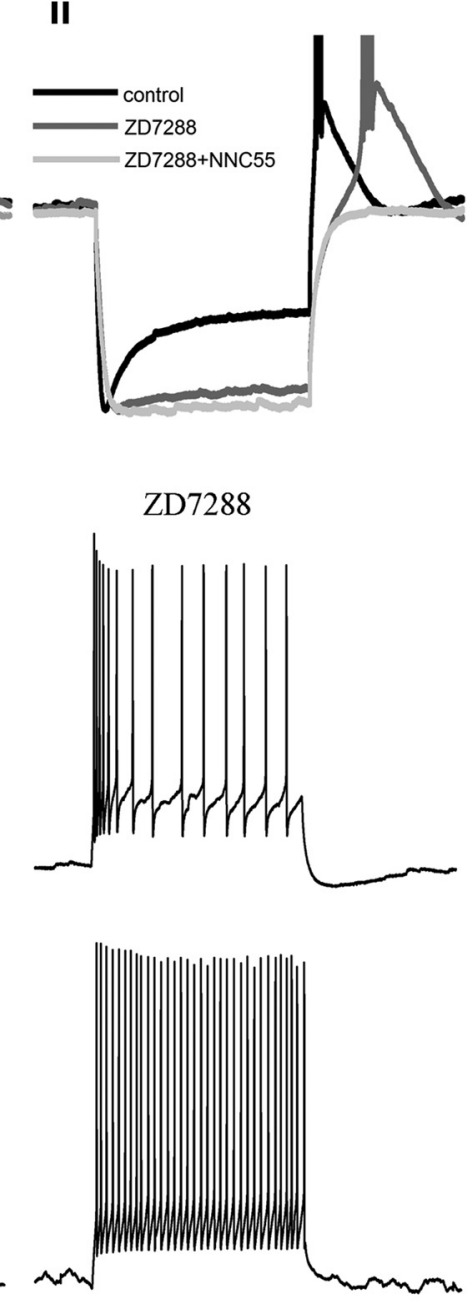

E
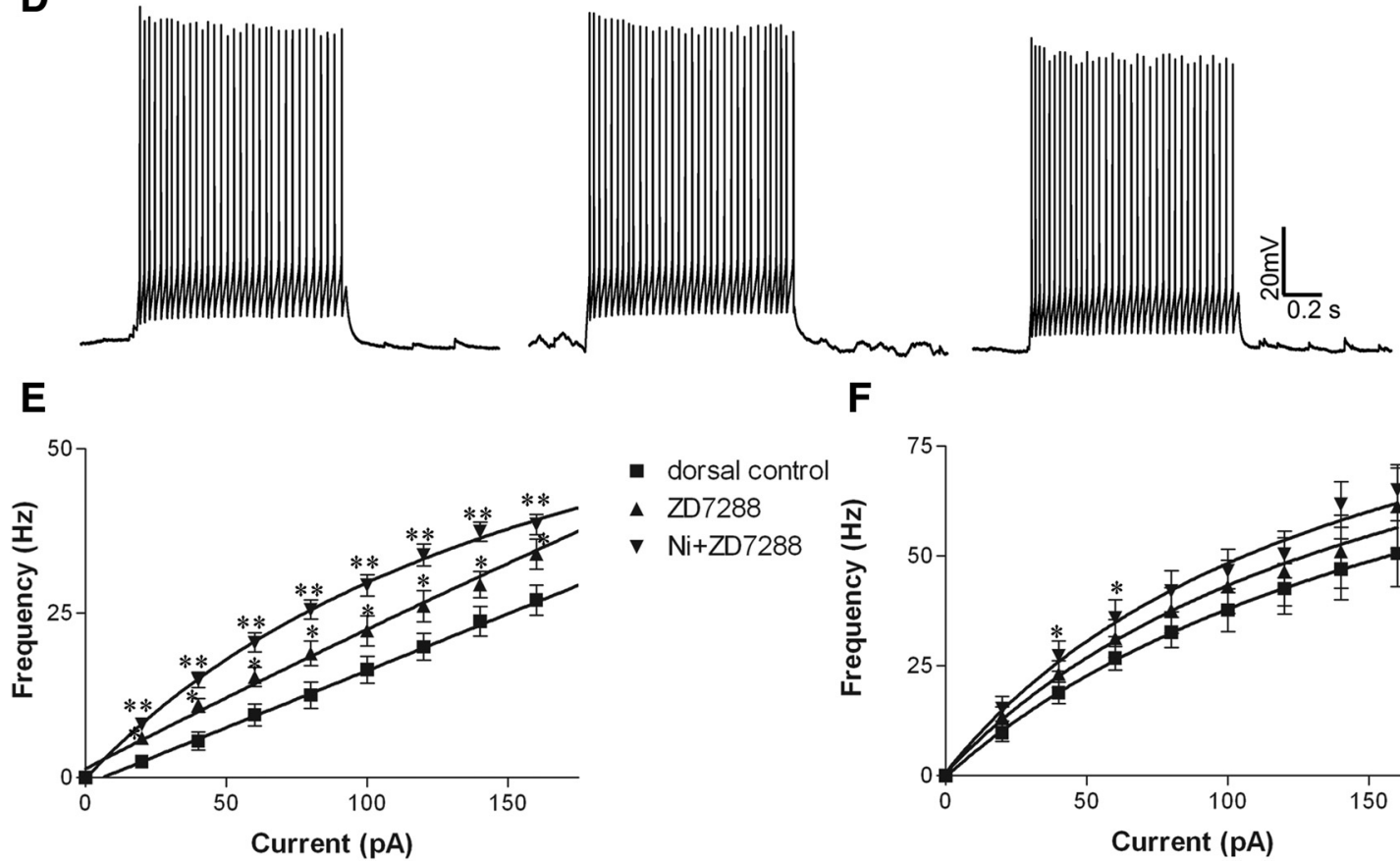

$\mathbf{F}$

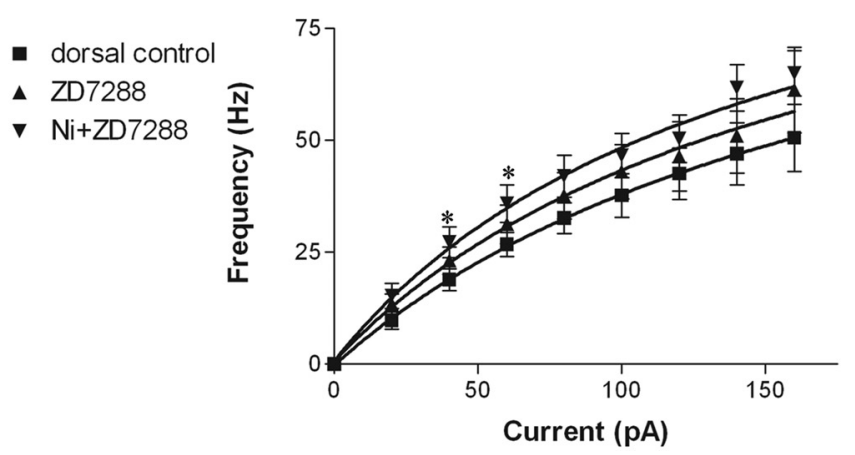

Figure 7. The effects of different channel blockers on the electrophysiological properties of V3 INs. A, Effects of ZD7288 (dark gray) and ZD7288 + nickel (light gray, Ai) or ZD7288 + NNC 55-0396 (Aii) on the sag voltage and rebound reactions of representative dorsal V3 INs from cluster 1. B, Effects of ZD7288 (dark gray) and ZD7288 + nickel (light gray) on cluster2. C, D, Effects of ZD7288 and ZD7288 + Ni on the spike patterns of representative cells from cluster $1(\boldsymbol{C})$ and cluster $2(\boldsymbol{D})$ responding to 1 s suprathreshold currents. $\boldsymbol{E}, \boldsymbol{F}, f-/$ plots of cells from cluster $1(\boldsymbol{E})$ and cluster $2(\boldsymbol{F}){ }^{*} p<0.05 ;{ }^{* *} p<0.01$.

$8 B, C)$. However, the number of activated dorsal V3 INs after swimming was comparable to controls, but the number was tripled after running (Fig. $8 B, C$ ). With the analysis of their confidence intervals, the increase of activated V3 INs at the ventral and intermediate regions after both running and swimming, and at the dorsal after running was significant compared with control situations, but there was no significant change for dorsal V3 INs after swimming (Fig. 8C). These results indicate that both dorsal and ventral V3 INs are actively recruited in running, while only ventral V3 INs are preferentially activated in swimming.

\section{Discussion}

In this study, we systematically analyzed the intrinsic properties of V3 spinal INs. Assisted by computational cluster analysis, we identified at least two subpopulations of V3 spinal INs, those located dorsally and ventrally in the mature spinal cord. These populations are not only spatially separate but are electrophysi- 
A
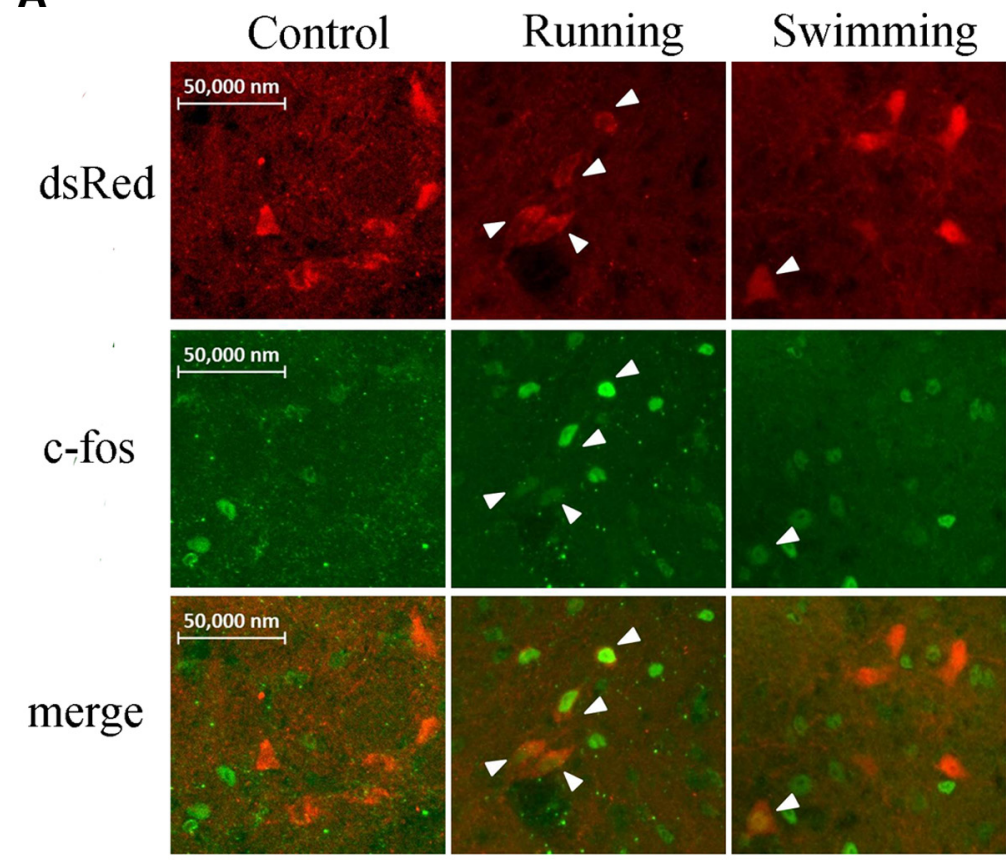

C

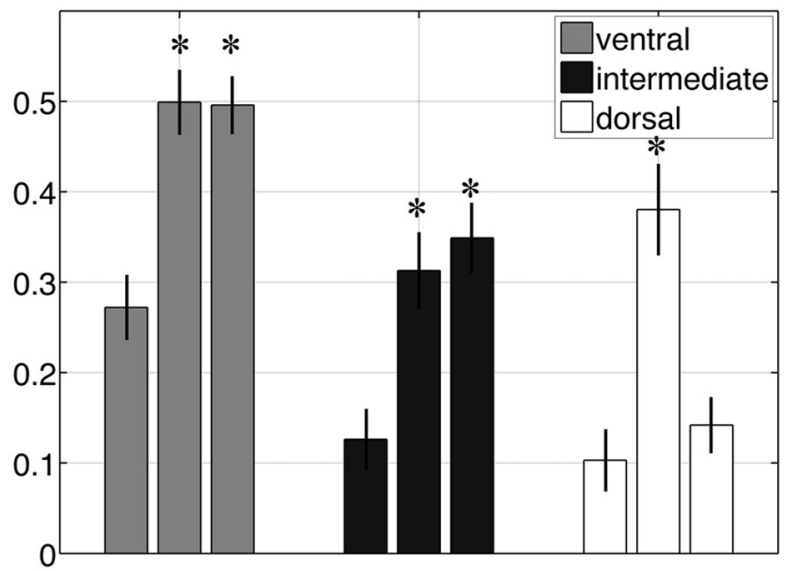

B
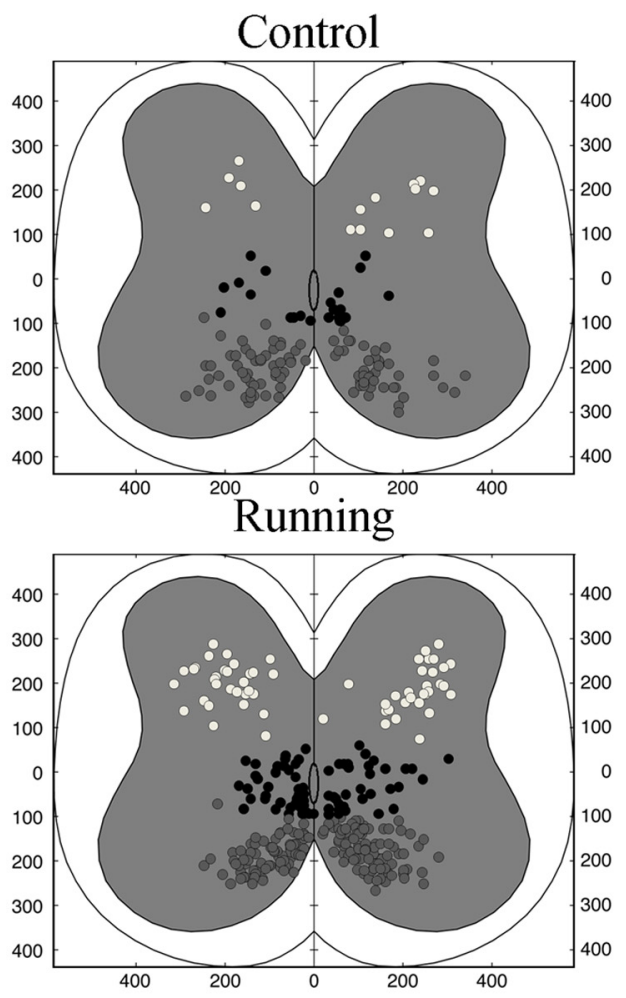

Swimming

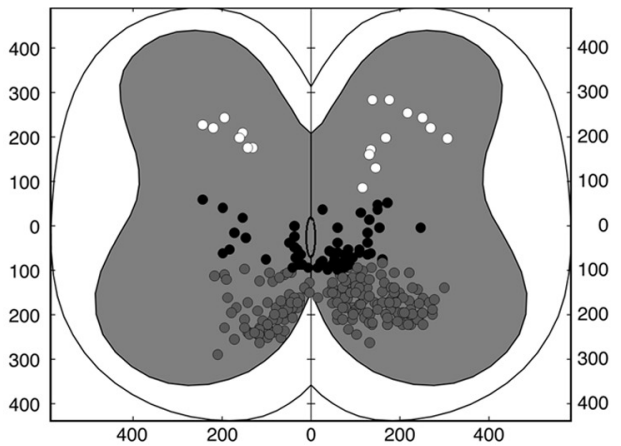

Figure 8. Differential recruitment of V3 INs in running and swimming behaviors. $\boldsymbol{A}$, Confocal image of dorsal V3 neurons (red) expressing c-Fos protein (green) at rest or after running and swimming. B, c-Fos-positive V3 INs are mapped onto cross sections of lumbar cords from control (Bi), running (Bii), and swimming (Biii) mice. Cells are divided into ventral (gray), intermediate (black), and dorsal (white). C, Histogram showing proportion of c-Fos-positive V3 INs relative to the total number V3 INs at different positions. ${ }^{*} p<0.01$.

ologically and morphologically distinct and differ in the roles that they play in locomotion. Our data also suggest that differential expression of ion channels may account for the unique electrophysiological properties displayed by these two groups of neurons.

\section{Two V3 subpopulations exist with distinct properties}

Electrophysiological and morphological properties determine how neurons respond to incoming signals and their function in networks, and they have been used as important criteria to classify functional subpopulations of neurons in the brain (Mel, 1994; Häusser et al., 2000; Yoshimura and Callaway, 2005; Ascoli et al., 2008; Graves et al., 2012). Although it is believed that spinal neurons are heterogeneous and exhibit different intrinsic properties, criteria that may be used to classify different neuronal subgroups based on these properties are still being established (Szûcs et al., 2003; Theiss and Heckman, 2005; Zhong et al.,
2006). Single-cell recordings from different spinal cord regions at various ages have yielded a mixture of response types, namely, cells exhibiting regular firing, bursting (chattering or phasic), delayed firing, or single spikes (Smith and Perrier, 2006; Dougherty and Kiehn, 2010; Zhong et al., 2010). However, no specific distribution patterns have been reported among these cells, and it is not clear how a particular pattern corresponds to a specific group of spinal neurons as defined by their genetic origin or synaptic connectivity.

Most of the studies in the literature describing the intrinsic properties of genetically defined INs in the mouse spinal cord have been undertaken in neonates (Wilson et al., 2005; Dougherty and Kiehn, 2010; Zhong et al., 2010). This study is one of the firsts to systematically investigate the intrinsic properties of genetically defined INs in functionally mature spinal cords. This is a key issue since the intrinsic properties of neurons change during postnatal development (Baccei and Fitzgerald, 2005; Walsh et al., 
2009; Tadros et al., 2012). In addition, mice establish mature locomotor behaviors in the first 3 weeks after birth. During this period of time, the CPGs with descending and peripheral inputs in the spinal cord gradually reach maturation (Clarac et al., 2004). Consistent with the other studies of mature spinal neurons in different model systems, we found that all recorded V3 INs generate constant repetitive spikes, even though V3 IN firing patterns appear relatively simple, and we only observed either tonic or adaptive spike patterns (Smith and Perrier, 2006; Husch et al., 2011).

The properties of individual V3 INs show great diversity, implying heterogeneity. Computational analyses provided us with an objective and unambiguous method to evaluate diverse properties of individual neurons simultaneously (Ma et al., 2006; Graves et al., 2012). Four attributes (capacitance, $f-I$ slope value, instantaneous spike frequency, and sag voltage) chosen by our cluster analysis program, served to classify V3 INs into two subgroups with distinct electrophysiological properties. These four attributes reflect different aspects of a neuron's intrinsic properties, from the size of its cell surface to its response to suprathreshold and subthreshold stimuli. The separation of these two V3 subpopulations is further confirmed by the fact that other properties, such as input resistance, adaptation rate, and s-AHP, also differed significantly between them.

Some other factors, such as post-inhibitory rebound potential and spikes, could not be measured in all cells and were thereby excluded from the model analysis. Nonetheless, they were also a signature feature that distinguished the two groups. We also did not include morphological properties in the cluster analyses, because we could only characterize total cell morphology in $\sim 50 \%$ of recorded cells. However, the morphological properties of the cells differed significantly between the assigned groups.

By using well known specific blockers for two types of voltagedependent ion channels, $I_{\mathrm{h}}$ and ICaT, we found that all V3 INs express a ZD7288-sensitive $I_{\mathrm{h}}$ current. Nonetheless, dorsal V3 INs have a larger sag voltage and a greater change in membrane properties after the $I_{\mathrm{h}}$ currents are blocked compared with ventral V3 INs. This suggests that dorsal V3 INs may express more $I_{\mathrm{h}}$ channels than ventral V3 cells. We have also shown that ICaT may be the primary ion current that produces strong rebound potentials in dorsal V3 INs, and that this current is less abundant in ventral cells. ICaT currents are likely to influence the firing properties in dorsal cells, and blocking both channels in dorsal V3 INs promotes more ventral V3-like characteristics.

In summary, our analyses allowed us to distinguish two V3 subpopulations: one is a high-gain group that has a uniform tonic firing pattern with simple morphology; while the other consists of low-gain V3 INs that exhibit diverse firing patterns and subthreshold properties, and complex spatial structures. These characteristics are likely to be important for their function in the motor circuitry.

\section{Functional roles of different V3 IN subpopulations}

Previous studies indicate that commissural V3 neurons function to establish stable gaits and synchronize outputs from the two halves of the spinal cord (Zhang et al., 2008; Rabe Bernhardt et al., 2012). We therefore asked whether two V3 subpopulations exhibiting unique properties played distinct roles in locomotion.

V3 INs are generated from the ventral-most progenitor domain, p3, adjacent to motor neuron progenitors (Zhang et al., 2008). Most V3 INs are excitatory commissural INs (CINs) clustered in the medial part of the spinal cord in lamina X, VIII, and VII and in the deep dorsal horns. Such profound spatial separa- tion may account for the differing participation of V3 INs in different networks. It has been shown that in adult cat and rodent spinal cords, ventral CINs (lamina VIII) receive intensive innervations from descending fibers and some group II afferents, dorsal CINs (laminae IV-V) receive group II and skin sensory afferents, and intermediate CINs (laminae VI-VII) are excited by multiple types of primary afferents (Jankowska, 2008; Jankowska and Edgley, 2010; Szokol et al., 2011). These results fit with our preliminary observation that dorsal and some intermediate V3 INs receive more sensory input than ventral V3 INs in the upper lumber cord. Our previous work (Geiman et al., 2006; Zhang et al., 2008) also indicated that V3 INs projected to a broad range of neurons in the ventral spinal cord. However, only ventral and some intermediate V3 INs connect directly to contralateral motor neurons. Our preliminary data have also shown, similar to other studies of spinal CINs (Stokke et al., 2002), that the axons of dorsal V3 INs ascend, while those of ventral and intermediate V3 INs either ascend or descend (Geiman et al., 2006, Blacklaws, 2013). As suggested by previous studies of excitatory CINs (Kiehn et al., 2008), such arrangements imply that ventral V3 INs are a group of premotor CINs responsible for synchronization of motor outputs at different levels across the lumbar spinal cord, while the dorsal and intermediate V3 INs function as sensory relay INs that may indirectly be involved in left-right coordination.

Such a prediction is supported by the different expression patterns of c-Fos protein after running and swimming. Our results show that ventral V3 INs are recruited in both activities, confirming their role as critical premotor neurons that are important for general locomotor behaviors, such as the coordinating the phase of limb movements (McCrea and Rybak, 2008). However, dorsal V3 INs function primarily in running rather than swimming. This result suggests that dorsal V3 INs might be more strongly activated by sensory feedback from cutaneous afferents that may be related to dynamic motor control and weight bearing. This would be consistent with previous studies showing that cutaneous input may play important roles in weight-bearing locomotion (Frigon and Rossignol, 2008; Sławińska et al., 2012).

The distinct physiological and morphological properties of $\mathrm{V} 3$ subpopulations could suit their functional roles well. For example, high-gain cells are mainly found within ventral and intermediate clusters, while dorsal V3 INs are all in the low-gain group. The steep $f-I$ slope, tonic firing characteristics, and relatively simple morphology would make ventral V3 INs sensitive to small input currents, thus ensuring that they faithfully mediate rapid communication among CPG neurons, descending neurons, and motor neurons. In addition, a wide range of gain values would allow ventral V3 INs to respond to given inputs with collective smooth outputs, avoiding sudden response surges in a particular direction. By contrast, dorsal V3 INs, as information-relaying neurons, have more complex dendritic structures and exhibit low input resistance, high rheobase values, and slow $f-I$ slopes with a large response range. These properties would enable them to respond in a graded manner to a variety of inputs. Interestingly, dorsal V3 INs show a mixture of firing patterns, from tonic to highly adaptive with initial bursts, potentially allowing them to filter and process incoming information in multiple modes. The large rebound potentials might also allow dorsal and intermediate V3 INs to play some role in generating or maintaining the locomotor rhythm. It is important to keep in mind that components of each physiologically defined subpopulation intermingle in the intermediate region. Although we predict that V3 INs in the intermediate region function in different circuits and that their intrinsic properties correlate with those functions, detailed 
connectivity and functional studies are required to distinguish the nature of $\mathrm{V} 3$ subtypes in this region.

In summary, our study reveals that the spatially separated ventral and dorsal V3 INs possess characteristic intrinsic property profiles, and that these distinct properties correlate with their recruitment during different locomotor activities. This is the first time that subgroups of spinal INs in adult mice have been shown to display specific intrinsic properties and topological positions that are associated with different locomotor functions. The results of our study open new avenues of investigation that will allow us to further probe the functional circuitry in the spinal cord using genetic tools combined with physiological and anatomical techniques.

\section{References}

Alvarez FJ, Benito-Gonzalez A, Siembab VC (2013) Principles of interneuron development learned from Renshaw cells and the motoneuron recurrent inhibitory circuit. Ann N Y Acad Sci 1279:22-31. CrossRef Medline

Arber S (2012) Motor circuits in action: specification, connectivity, and function. Neuron 74:975-989. CrossRef Medline

Ascoli GA, Alonso-Nanclares L, Anderson SA, Barrionuevo G, BenavidesPiccione R, Burkhalter A, Buzsáki G, Cauli B, Defelipe J, Fairén A, Feldmeyer D, Fishell G, Fregnac Y, Freund TF, Gardner D, Gardner EP, Goldberg JH, Helmstaedter M, Hestrin S, Karube F, et al. (2008) Petilla terminology: nomenclature of features of GABAergic interneurons of the cerebral cortex. Nat Rev Neurosci 9:557-568. CrossRef Medline

Baccei ML, Fitzgerald M (2005) Intrinsic firing properties of developing rat superficial dorsal horn neurons. Neuroreport 16:1325-1328. CrossRef Medline

Blacklaws J (2013) The development of V3 interneurons in the mouse spinal cord. PhD thesis, Dalhousie University.

Briscoe J, Sussel L, Serup P, Hartigan-O'Connor D, Jessell TM, Rubenstein JL, Ericson J (1999) Homeobox gene Nkx2.2 and specification of neuronal identity by graded Sonic hedgehog signalling. Nature 398:622-627. CrossRef Medline

Cazalets JR, Sqalli-Houssaini Y, Clarac F (1992) Activation of the central pattern generators for locomotion by serotonin and excitatory amino acids in neonatal rat. J Physiol 455:187-204. Medline

Clarac F, Brocard F, Vinay L (2004) The maturation of locomotor networks. Prog Brain Res 143:57-66. CrossRef Medline

Dai X, Noga BR, Douglas JR, Jordan LM (2005) Localization of spinal neurons activated during locomotion using the $\mathrm{c}$-fos immunohistochemical method. J Neurophysiol 93:3442-3452. CrossRef Medline

Dougherty KJ, Kiehn O (2010) Firing properties and cellular properties of V2a interneurons in the rodent spinal cord. J Neurosci 30:24-37. CrossRef Medline

Dyck J, Lanuza GM, Gosgnach S (2012) Functional characterization of dI6 interneurons in the neonatal mouse spinal cord. J Neurophysiol 107: 3256-3266. CrossRef Medline

Frigon A, Rossignol S (2008) Adaptive changes of the locomotor pattern and cutaneous reflexes during locomotion studied in the same cats before and after spinalization. J Physiol 586:2927-2945. CrossRef Medline

Geiman E, Zhang Y, Narayan S, Lanuza, Goulding M (2006) Anatomical characterization of $\mathrm{V} 3$ interneuron subpopulations in the neonatal mouse spinal cord. Soc Neurosci Abstr 32:252.10.

Goulding M (2009) Circuits controlling vertebrate locomotion: moving in a new direction. Nat Rev Neurosci 10:507-518. CrossRef Medline

Graves AR, Moore SJ, Bloss EB, Mensh BD, Kath WL, Spruston N (2012) Hippocampal pyramidal neurons comprise two distinct cell types that are countermodulated by metabotropic receptors. Neuron 76:776-789. CrossRef Medline

Grillner S, Wallén P (1985) Central pattern generators for locomotion, with special reference to vertebrates. Annu Rev Neurosci 8:233-261. CrossRef Medline

Hastie T, Tibshirani R, Friedman J (2009) The elements of statistical learning: data mining, inference, and prediction, Ed 2. New York: Springer.

Häusser M, Spruston N, Stuart GJ (2000) Diversity and dynamics of dendritic signaling. Science 290:739-744. CrossRef Medline

Huang L, Keyser BM, Tagmose TM, Hansen JB, Taylor JT, Zhuang H, Zhang M, Ragsdale DS, Li M (2004) NNC 55-0396 [(1S,2S)-2-(2-(N-[(3-benzimida-
zol-2-yl)propyl]-N-methylamino)ethyl)-6-fluoro-1,2,3,4-tetrahydro-1isopropyl-2-naphtyl cyclopropanecarboxylate dihydrochloride]: a new selective inhibitor of T-type calcium channels. J Pharmacol Exp Ther 309: 193-199. CrossRef Medline

Husch A, Cramer N, Harris-Warrick RM (2011) Long-duration perforated patch recordings from spinal interneurons of adult mice. J Neurophysiol 106:2783-2789. CrossRef Medline

Jankowska E (2001) Spinal interneuronal systems: identification, multifunctional character and reconfigurations in mammals. J Physiol 533:3140. CrossRef Medline

Jankowska E (2008) Spinal interneuronal networks in the cat: elementary components. Brain Res Rev 57:46-55. CrossRef Medline

Jankowska E, Edgley SA (2010) Functional subdivision of feline spinal interneurons in reflex pathways from group Ib and II muscle afferents; an update. Eur J Neurosci 32:881-893. CrossRef Medline

Kiehn O, Kullander K (2004) Central pattern generators deciphered by molecular genetics. Neuron 41:317-321. CrossRef Medline

Kjaerulff O, Kiehn O (1996) Distribution of networks generating and coordinating locomotor activity in the neonatal rat spinal cord in vitro: a lesion study. J Neurosci 16:5777-5794. Medline

Ma Y, Hu H, Berrebi AS, Mathers PH, Agmon A (2006) Distinct subtypes of somatostatin-containing neocortical interneurons revealed in transgenic mice. J Neurosci 26:5069-5082. CrossRef Medline

Madisen L, Zwingman TA, Sunkin SM, Oh SW, Zariwala HA, Gu H, Ng LL, Palmiter RD, Hawrylycz MJ, Jones AR, Lein ES, Zeng H (2010) A robust and high-throughput Cre reporting and characterization system for the whole mouse brain. Nat Neurosci 13:133-140. CrossRef Medline

McCrea DA, Rybak IA (2008) Organization of mammalian locomotor rhythm and pattern generation. Brain Res Rev 57:134-146. CrossRef Medline

McDonough SI, Bean BP (1998) Mibefradil inhibition of T-type calcium channels in cerebellar purkinje neurons. Mol Pharmacol 54:1080-1087. Medline

Mel BW (1994) Information processing in dendritic trees. Neural Comput 6:1031-1085. CrossRef

Rabe Bernhardt N, Memic F, Gezelius H, Thiebes AL, Vallstedt A, Kullander K (2012) DCC mediated axon guidance of spinal interneurons is essential for normal locomotor central pattern generator function. Dev Biol 366:279-289. CrossRef Medline

Renshaw B (1941) Influence of the discharge of motoneurons upon exciation of neighboring motoneurons. J Neurophysiol 4:167-183.

Renshaw B (1946) Central effects of centripetal impulses in axons of spinal ventral roots. J Neurophysiol 9:191-204. Medline

Sławińska U, Majczyński H, Dai Y, Jordan LM (2012) The upright posture improves plantar stepping and alters responses to serotonergic drugs in spinal rats. J Physiol 590:1721-1736. CrossRef Medline

Smith M, Perrier JF (2006) Intrinsic properties shape the firing pattern of ventral horn interneurons from the spinal cord of the adult turtle. J Neurophysiol 96:2670-2677. CrossRef Medline

Stam FJ, Hendricks TJ, Zhang J, Geiman EJ, Francius C, Labosky PA, Clotman F, Goulding M (2012) Renshaw cell interneuron specialization is controlled by a temporally restricted transcription factor program. Development 139:179-190. CrossRef Medline

Stokke MF, Nissen UV, Glover JC, Kiehn O (2002) Projection patterns of commissural interneurons in the lumbar spinal cord of the neonatal rat. J Comp Neurol 446:349-359. CrossRef Medline

Sugimori M, Nagao M, Bertrand N, Parras CM, Guillemot F, Nakafuku M (2007) Combinatorial actions of patterning and HLH transcription factors in the spatiotemporal control of neurogenesis and gliogenesis in the developing spinal cord. Development 134:1617-1629. CrossRef Medline

Szokol K, Glover JC, Perreault MC (2011) Organization of functional synaptic connections between medullary reticulospinal neurons and lumbar descending commissural interneurons in the neonatal mouse. J Neurosci 31:4731-4742. CrossRef Medline

Szûcs P, Odeh F, Szokol K, Antal M (2003) Neurons with distinctive firing patterns, morphology and distribution in laminae V-VII of the neonatal rat lumbar spinal cord. Eur J Neurosci 17:537-544. CrossRef Medline

Tadros MA, Harris BM, Anderson WB, Brichta AM, Graham BA, Callister RJ (2012) Are all spinal segments equal: intrinsic membrane properties of 
superficial dorsal horn neurons in the developing and mature mouse spinal cord. J Physiol 590:2409-2425. CrossRef Medline

Theiss RD, Heckman CJ (2005) Systematic variation in effects of serotonin andnorepinephrine on repetitive firing properties of ventral horn neurons. Neuroscience 134:803-815. CrossRef Medline

Walsh MA, Graham BA, Brichta AM, Callister RJ (2009) Evidence for a critical period in the development of excitability and potassium currents in mouse lumbar superficial dorsal horn neurons. J Neurophysiol 101: 1800-1812. CrossRef Medline

Wilson JM, Hartley R, Maxwell DJ, Todd AJ, Lieberam I, Kaltschmidt JA, Yoshida Y, Jessell TM, Brownstone RM (2005) Conditional rhythmicity of ventral spinal interneurons defined by expression of the $\mathrm{Hb} 9$ homeodomain protein. J Neurosci 25:5710-5719. CrossRef Medline

Yoshimura Y, Callaway EM (2005) Fine-scale specificity of cortical net- works depends on inhibitory cell type and connectivity. Nat Neurosci 8:1552-1559. CrossRef Medline

Zhang Y, Narayan S, Geiman E, Lanuza GM, Velasquez T, Shanks B, Akay T, Dyck J, Pearson K, Gosgnach S, Fan CM, Goulding M (2008) V3 spinal neurons establish a robust and balanced locomotor rhythm during walking. Neuron 60:84-96. CrossRef Medline

Zhong G, Díaz-Ríos M, Harris-Warrick RM (2006) Intrinsic and functional differences among commissural interneurons during fictive locomotion and serotonergic modulation in the neonatal mouse. J Neurosci 26:65096517. CrossRef Medline

Zhong G, Droho S, Crone SA, Dietz S, Kwan AC, Webb WW, Sharma K, Harris-Warrick RM (2010) Electrophysiological characterization of V2a interneurons and their locomotor-related activity in the neonatal mouse spinal cord. J Neurosci 30:170-182. CrossRef Medline 